\title{
Scalings for unsteady natural convection boundary layers on an evenly heated plate with time-dependent heating flux
}

\author{
Wenxian $\operatorname{Lin}^{1,2, *}$ and S. W. Armfield ${ }^{3}$ \\ ${ }^{1}$ Solar Energy Research Institute, Yunnan Normal University, Kunming, Yunnan 650092, People’s Republic of China \\ ${ }^{2}$ School of Engineering, James Cook University, Townsville, QLD 4811, Australia \\ ${ }^{3}$ School of Aerospace, Mechanical and Mechatronic Engineering, University of Sydney, NSW 2006, Australia
}

(Received 29 July 2013; published 18 December 2013)

\begin{abstract}
It is of fundamental significance, especially with regard to application, to fully understand the flow behavior of unsteady natural convection boundary layers on a vertical plate heated by a time-dependent heat flux. Such an understanding is currently scarce. In this paper, the scaling analysis by Lin et al. [Phys. Rev. E 79, 066313 (2009)] using a simple three-region structure for the unsteady natural convection boundary layer of a homogeneous Newtonian fluid with $\operatorname{Pr}>1$ under isothermal heating was substantially extended for the case when the heating is due to a time-varying sinusoidal heat flux. A series of scalings was developed for the thermal boundary thickness, the plate temperature, the viscous boundary thicknesses, and the maximum vertical velocity within the boundary layer, which are the major parameters representing the flow behavior, in terms of the governing parameters of the flow, i.e., the Rayleigh number Ra, the Prandtl number Pr, and the dimensionless natural frequency $f_{n}$ of the time-varying sinusoidal heat flux, at the start-up stage, at the transition time scale which represents the ending of the start-up stage and the beginning of the transitional stage of the boundary-layer development, and at the quasi-steady stage. These scalings were validated by comparison to 10 full numerical solutions of the governing equations with $\mathrm{Ra}, \operatorname{Pr}$, and $f_{n}$ in the ranges $10^{6} \leqslant \mathrm{Ra} \leqslant 10^{9}, 3 \leqslant \operatorname{Pr} \leqslant 100$, and $0.01 \leqslant f_{n} \leqslant 0.1$ and were shown in general to provide an accurate description of the flow at different development stages, except for high-Pr runs in which a further, although weak, Pr dependence is present, which cannot be accurately predicted by the current scaling analysis using the simple three-region structure, attributed to the non-boundary-layer nature of the velocity field with high-Pr fluids. Some scalings at the transition time scale and at the quasi-steady stage also produce noticeable deviations from the numerical results when $f_{n}$ is reduced, indicating that there may be a further $f_{n}$ dependence of the scalings which also cannot be accurately predicted by the current scaling analysis.
\end{abstract}

DOI: 10.1103/PhysRevE.88.063013

PACS number(s): 44.25.+f, 47.55.P-, 44.20.+b, 47.15.Cb

\section{INTRODUCTION}

Scaling analysis has proven to be a very effective tool to reveal the transient behavior of unsteady natural convection flow since Patterson and Imberger [1] carried out a pioneering scaling analysis of the transient natural convection boundarylayer flow in a two-dimensional rectangular cavity with differentially heated sidewalls. Numerous subsequent studies have extended the scaling analysis to many different aspects of transient natural convection boundary layers under various configurations and flow conditions. The readers are referred to our recent paper [2] for a detailed review of some of these studies.

Unsteady natural convection boundary layers on a vertical plate heated by a time-dependent heat flux are found in many applications, such as in the Trombe wall system of a passive solar house and in a solar chimney for electricity generation (see, e.g., Refs. [3-8]). In the Trombe wall case, the wall, which is usually painted black or with a solar selective coating, absorbs solar radiation and converts it into heat, which is then transported to the dwelling by the heated air via a natural convection boundary-layer flow in the channel formed by the glazing and the wall. A solar chimney operates in a similar manner. For both cases, the time-dependent solar radiation, which varies sinusoidally under a clear sky condition (only in

*wenxian.lin@jcu.edu.au the first half of the sinusoidal cycle), serves as the heat flux for the natural convection boundary-layer flows.

Although there have been numerous studies on natural convection boundary layers on a vertical plate heated by a heat flux, the majority of these studies have been on the cases where the applied heat flux is either uniformly constant or spatially varied but not time dependent.

In the uniformly constant heat flux case, for example, Cheng [9] obtained solutions for the steady natural convection heat transfer in a saturated porous medium adjacent to heated surfaces with a prescribed constant heat flux based on the boundary-layer approximations. Carey and Mollendorf [10] measured variations of thermal boundary-layer thickness with $\mathrm{Pr}$ in the range from 0.703 (air) to 8940 (silicone oil) for the steady natural convection from a vertical uniformheat-flux surface and found that their experimental results were in general in very good agreement with the similarity solutions obtained by Sparrow and Gregg [11], although large disagreement was also observed for the highest-Pr fluid, which was attributed to the non-boundary-layer nature of the velocity field. Bejan [12] solved the steady natural convection boundary-layer flow in a fluid-saturated porous media with a prescribed uniform heat flux. Bark, Alavyoon, and Dahlkild [13] carried out a theoretical study on the unsteady natural convection boundary-layer flow in a vertical slot driven by prescribed fluxes of heat or mass at the vertical walls of the slot and showed that the boundary-layer thicknesses scale with $\mathrm{Ra}^{1 / 5}$, in good agreement with the available experimental results. Alavyoon [14] extended this 
study to the unsteady double-diffusive natural convection in a rectangular fluid-saturated vertical porous enclosure subject to prescribed fluxes of heat and mass for a wide range of the Rayleigh-Darcy number, inverse of buoyancy ratio, Lewis number, and enclosure aspect ratio. Imadojemu and Johnson [15] obtained experimentally the correlation between the Nusselt number and $\mathrm{Ra}$ for the natural convection boundarylayer flow on a vertical plate in water heated by a constant heat flux with $\mathrm{Ra}$ in the range from $10^{4}$ to $10^{13}$. Sundström and Vynnycky [16] performed a linear stability analysis of natural convection flows in an arbitrarily inclined rectangular enclosure driven by prescribed heat or mass fluxes along two opposing walls. Aydin and Guessous [17] carried out a dimensional analysis to develop fundamental correlations for laminar and turbulent natural convection from a uniformly heated vertical plate and introduced a dimensionless parameter to quantify the relevant heat transfer characteristics, which were found to be dependent on $\mathrm{Pr}$ and the modified Ra. Armfield, Patterson, and Lin [18] carried out a scaling analysis to obtain the scalings for the unsteady natural convection boundary layer on an evenly heated semi-infinite plate with isoflux heating in ambient fluids with $\operatorname{Pr}>1$, whereas Lin, Armfield, and Patterson [19] developed the scaling for the similar flow configuration but with $\operatorname{Pr}<1$ fluids. Bednarz, Lei, and Patterson [20] conducted a numerical study on the unsteady natural convection induced by isoflux surface cooling in a reservior model and examined the flow response to random perturbations of different amplitudes. Following a similarity analysis of the transport equations, Khan and Aziz [21] investigated numerically the steady natural convection flow of a nanofluid over a vertical plate with a constant surface heat flux and showed that velocity, temperature, and concentration profiles in the respective boundary layers depend on the Prandtl and Lewis numbers, a Brownian motion parameter, a thermophoresis parameter, and a buoyancy ratio parameter. Capobianchi and Aziz [22] conducted a scaling analysis for the natural convection flow on a vertical plate under a constant surface heat flux. Saha, Brown, and Gu [23] obtained the scalings via scaling analysis for the unsteady natural convection boundary layer adjacent to a downward-facing inclined plate with a uniform heat flux. Buonomo and Manca [24] carried out a numerical study on the transient natural convection in a vertical microchannel heated at a uniform heat flux and proposed a composite correlation to estimate average Nusselt number in terms of $\mathrm{Ra}$ and the Knudsen number. Merkin [25] obtained similarity solutions for steady natural convection boundary-layer flow in a heat-generating porous medium with a constant surface heat flux.

There have also been some studies on the natural convection boundary-layer flows on a vertical plate heated by spatially varied but time-independent surface fluxes. For example, Dutta and Seetharamu [26] obtained solutions for steady natural convection in a saturated porous medium adjacent to a vertical impermeable wall subjected to a nonuniform heat flux that is an arbitrary function of the distance along the surface. Kou and Huang [27] developed similarity solutions for the steady natural convection boundary-layer flows on a vertical wall embedded in porous media with prescribed power-law and exponential heat fluxes that vary with the location on the plate, and Wright, Ingham, and Pop [28] carried out a similar study but with a different form of the prescribed power-law heat flux. Ganesan and Rani [29] studied numerically the unsteady natural convection boundary-layer flow over a semi-infinite vertical cylinder subjected to power-law surface heat and mass fluxes. A numerical study was also conducted by Tashtoush and Abu-Irshaid [30] for the steady natural convection flow from a wavy surface subjected to a power-law variable heat flux and it was found that the wavelength of the local Nusselt number and surface temperature variation was equal to the wavy surface but the average Nusselt number was only half of the wavy surface. Similarly, Shalini and Kumar [31] undertook a numerical investigation of a power-law variable heat flux for the steady natural convection flow along a corrugated vertical wall in porous media. Tsai and Huang [32] obtained the solutions for heat and mass transfer from steady natural convection flow along a vertical surface with variable heat fluxes embedded in a porous medium due to thermal-diffusion (Soret) and diffusion-thermo (Dufour) effects. Mustafa, Asghar, and Hossain [33] studied the effects of steady natural convection flow of a viscoelistic second-grade fluid along vertical plate with a spatially varied heat flux. Palani and Kim [34] carried out a numerical study to examine the heat transfer characteristics of the unsteady natural convection past a vertical cone subjected to variable surface heat flux under the combined effects of magnetic field and thermal radiation.

Although important due to the significance in applications, the studies on the unsteady natural convection boundary layers on a vertical plate heated by a time-dependent heat flux are scarce. To our best knowledge, only a few relevant studies exist in the literature. One study was done by Fohr and Moussa [35], who carried out an experimental and numerical study on the heat and mass transfer in a cylindrical grain silo driven by unsteady natural convection boundary-layer flow when the silo was submitted to a time-dependent periodical wall heat flux which represented the periodic and meteorologic influences of solar radiation, ambient air temperature, and wind. They identified two distinct flow configurations: During the first half period, when the heat flux is high, the main part of the heat and mass transfer takes place in the natural convection boundary layer at the wall and this layer is fed with fresh air from the bottom and from the top through a central aspiration; during the second half period, when the heat flow is low, the stored heat provides a convective flux of a chimney type. Another study was carried out by Wang, Zeng, and Wang [36], who conducted a numerical study on the three-dimensional unsteady natural convections in an inclined porous cavity with time-dependent sinusoidal oscillating temperature boundary conditions and examined the combination effects of inclination angles and temperature oscillation frequency on the natural convection characteristics with $\mathrm{Ra}=10^{6}$ and $10^{7}$. They also carried out experimental measurements of the transient heat flux for the natural convection in an inclined enclosure with time-periodically varying wall temperature [37].

From the literature analysis, it is evident that our current understanding of the unsteady natural convection boundary layers on a vertical plate heated by a time-dependent heat flux, in particular quantifying the flow dynamics and heat transfer characteristics in terms of the governing parameters such as $\mathrm{Ra}$, $\mathrm{Pr}$, and the frequency of the time-periodically varying heating flux on the plate, is lacking. This motivates the current study to 
use scaling analysis to develop scalings for the unsteady natural convection boundary layer of a homogeneous Newtonian fluid with $\operatorname{Pr}>1$ adjacent to a vertical plate evenly heated with a time-varying sinusoidal heat flux and to carry out a series of direct numerical simulation (DNS) to validate the developed scalings.

The remainder of this paper is organized as follows. The scaling analysis is carried out in Sec. II to develop the scalings for the flow. These scalings and the governing equations are made dimensionless in Sec. III and then validated and analyzed with a series of DNS results in Sec. IV. Finally, conclusions are made in Sec. V.

\section{SCALINGS}

Under consideration is the unsteady natural convection boundary layer of a homogeneous Newtonian fluid with $\mathrm{Pr}>1$ adjacent to a vertical plate of height $H(\mathrm{~m})$ evenly heated with a time-varying sinusoidal heat flux in the form of

$$
\frac{d T}{d X}=-\Gamma_{w}(t)=-\Gamma_{w m} \sin (2 \pi f t),
$$

where $T\left({ }^{\circ} \mathrm{C}\right)$ is temperature, $X(\mathrm{~m})$ is the horizontal coordinate, $\Gamma_{w}(t)\left({ }^{\circ} \mathrm{C} / \mathrm{m}\right)$ is the transient temperature gradient across at the plate at time instant $t(\mathrm{~s}), \Gamma_{w m}\left({ }^{\circ} \mathrm{C} / \mathrm{m}\right)$ is the maximum temperature gradient across at the plate, and $f(\mathrm{~Hz})$ is the natural frequency of the time-varying flux applied to the plate. The flow is assumed to be two dimensional and the fluid is initially at rest. The plate lies at $X=0$ with the origin at $Y=0[Y(\mathrm{~m})$ is the vertical coordinate $]$, with the plate boundary conditions

$$
\begin{aligned}
U & =V=0, \\
\frac{d T}{d X} & =x-\Gamma_{w m} \sin (2 \pi f t) \text { at } x=0 \text { for } Y>0, \quad t \geqslant 0,
\end{aligned}
$$

where $\Gamma_{w m}$ and $f$ are assumed to be constant for a specific time-varying flux condition.

The governing equations of motion are the Navier-Stokes equations with the Boussinesq approximation for buoyancy, which together with the temperature equation can be written in the following two-dimensional forms:

$$
\begin{gathered}
\frac{\partial U}{\partial X}+\frac{\partial V}{\partial Y}=0 \\
\frac{\partial U}{\partial t}+\frac{\partial(U U)}{\partial X}+\frac{\partial(V U)}{\partial Y}=-\frac{1}{\rho} \frac{\partial P}{\partial X}+v\left(\frac{\partial^{2} U}{\partial X^{2}}+\frac{\partial^{2} U}{\partial Y^{2}}\right),
\end{gathered}
$$

$$
\begin{aligned}
& \frac{\partial V}{\partial t}+\frac{\partial(U V)}{\partial X}+\frac{\partial(V V)}{\partial Y} \\
&=-\frac{1}{\rho} \frac{\partial P}{\partial Y}+v\left(\frac{\partial^{2} V}{\partial X^{2}}+\frac{\partial^{2} V}{\partial Y^{2}}\right)+g \beta\left(T-T_{a}\right) \\
& \frac{\partial T}{\partial t}+\frac{\partial(U T)}{\partial X}+\frac{\partial(V T)}{\partial Y}=\kappa\left(\frac{\partial^{2} T}{\partial X^{2}}+\frac{\partial^{2} T}{\partial Y^{2}}\right)
\end{aligned}
$$

where $U(\mathrm{~m} / \mathrm{s})$ and $V(\mathrm{~m} / \mathrm{s})$ are the horizontal ( $X$ direction) and vertical ( $Y$ direction) velocity components; $P(\mathrm{~Pa})$ is pressure, $g\left(\mathrm{~m} / \mathrm{s}^{2}\right)$ is the acceleration due to gravity; $T_{a}\left({ }^{\circ} \mathrm{C}\right)$ is the initial temperature of the ambient fluid; and $\beta(1 / \mathrm{K})$, $v\left(\mathrm{~m}^{2} / \mathrm{s}\right)$, and $\kappa\left(\mathrm{m}^{2} / \mathrm{s}\right)$ are the thermal expansion coefficient, kinematic viscosity, and thermal diffusivity of the fluid at the temperature $T_{a}$, respectively. Gravity acts in the negative $Y$ direction.

For the unsteady natural convection boundary-layer flow considered here, the major governing parameters are the Rayleigh number Ra and the Prandtl number Pr, defined as

$$
\operatorname{Pr}=\frac{v}{\kappa}, \quad \operatorname{Ra}=\frac{g \beta \bar{\Gamma}_{w} H^{4}}{\nu \kappa},
$$

where $\bar{\Gamma}_{w}\left({ }^{\circ} \mathrm{C} / \mathrm{m}\right)$ is the time-averaged temperature gradient across the plate thickness which is calculated by

$$
\bar{\Gamma}_{w}=\frac{1}{t_{\text {total }}} \int_{0}^{t_{\text {total }}} \Gamma_{w m} \sin (2 \pi f t) d t=\frac{2}{\pi} \Gamma_{w m},
$$

in which $t_{\text {total }}(\mathrm{s})$ is the total heating time of the time-varying flux applied to the plate. In this paper, it is assumed that $2 \pi f t_{\text {total }}=\pi$, i.e., $f=0.5 / t_{\text {total }}$ (hence, only the first half, heating cycle, is considered, which is in line with the timedependent solar radiation model under a clear sky condition, although the scalings developed here are not limited to the cases driven by time-dependent solar radiation). Apparently, Ra defined above is the time-averaged global Rayleigh number for the unsteady natural convection boundary layer over the duration of heating under the time-varying flux applied to the plate, which is calculated in terms of $\Gamma_{w m}$ by

$$
\mathrm{Ra}=\left(\frac{2}{\pi}\right) \frac{g \beta \Gamma_{w m} H^{4}}{\nu \kappa} .
$$

The vertical boundary layer that develops adjacent to the plate after the initiation of the flow will experience a start-up stage dominated by one-dimensional conduction, a transitional stage involving traveling waves caused by the leading edge effect and a transition to two-dimensional convection, and eventually a quasi-steady state [38-40]. This differs slightly from the cases when the heating conditions are constant, such as the constant isothermal heating case studied by Ref. [41] where the boundary layer development mainly involves two dominant stages, i.e., a start-up stage similar to the current case and a steady state when the development becomes independent of time. In the current case, however, as the heating condition varies continuously with time only a quasi-steady state can be attained at the later stage of the development. The division between the start-up stage and the transitional stage is represented by the transition time scale $t_{s}\left(\mathrm{~s}, \tau_{s}\right.$ is its dimensionless form), as illustrated in Fig. 1 where a typical numerically simulated time series of the thermal boundary-layer thickness $\Delta_{T}\left(\mathrm{~m}, \delta_{T}=\Delta_{T} / H\right.$ is its dimensionless form) at height $Y=0.5 \mathrm{H}$ for the specific case $\operatorname{Ra}=10^{8}, \operatorname{Pr}=7$, and $f_{n}=0.1$ is shown. $\Delta_{T}$ is defined as the horizontal distance between the plate and the location where the fluid temperature reaches $0.01\left(T_{w}-T_{a}\right)$, where $T_{w}$ $\left({ }^{\circ} \mathrm{C}\right)$ is the plate temperature at height $Y$. Similar behavior is also observed for the other parameters of interest to this work, i.e., the plate temperature, $T_{w}$, the maximum vertical velocity within the boundary layer, $V_{m}(\mathrm{~m} / \mathrm{s})$, the inner viscous 


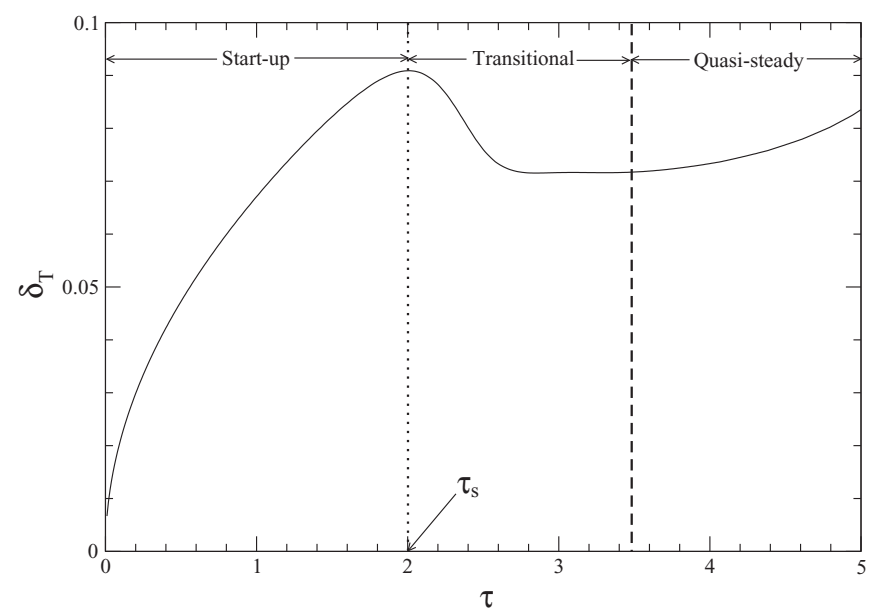

FIG. 1. The three distinct stages in the boundary-layer development, seen in the typical numerically simulated time series of the dimensionless local thermal boundary-layer thickness $\delta_{T}=\Delta_{T} / H$ at height $Y=0.5 H$ for the specific case $\operatorname{Ra}=10^{8}, \operatorname{Pr}=7$ and $f_{n}=0.1$, where time $\tau$ is made dimensonless by $H / V_{0}, f_{n}$ is the dimensionless natural frequency of the time-varying flux applied to the plate as defined by (43), and $\tau_{s}\left(=t_{s} /\left(H / V_{0}\right)\right)$ is the dimensionless transition time scale representing the end of the start-up stage and the beginning of the transitional stage.

boundary-layer thickness, $\Delta_{v i}(\mathrm{~m})$, and the outer viscous boundary-layer thickness, $\Delta_{v}(\mathrm{~m})$.

The scaling analysis is carried out by examining in detail the various balances in the governing equations by following the same procedures as used by Ref. [41] for the constant isothermal heating case and employing the same three-region structure originally proposed by Refs. [41,42], as depicted by Fig. 1 in Ref. [41], but modified and expanded appropriately by taking into account of the time-varying nature of the flux applied to the plate. To minimize the duplication of Ref. [41], only new and modified or expanded steps and equations of the scaling analysis will be presented below for the time-varying flux case studied by this work. The readers are referred to Ref. [41] for the details that are the same as the current case.

\section{A. Scalings at the start-up stage}

At the start-up stage, it is found that the scalings for $\Delta_{T}$, $\Delta_{v i}, \Delta_{v}$, and $V_{m}$ have the same forms as those obtained in Ref. [41] for the constant isothermal heating case, i.e.,

$$
\begin{gathered}
\Delta_{T} \sim \kappa^{\frac{1}{2}} t^{\frac{1}{2}} \\
\Delta_{v i} \sim \frac{1}{1+\operatorname{Pr}^{-\frac{1}{2}}} \Delta_{T} \sim \frac{1}{1+\operatorname{Pr}^{-\frac{1}{2}}} \kappa^{\frac{1}{2}} t^{\frac{1}{2}}, \\
\Delta_{v} \sim \operatorname{Pr}^{\frac{1}{2}} \Delta_{T} \sim \operatorname{Pr}^{\frac{1}{2}} \kappa^{\frac{1}{2}} t^{\frac{1}{2}} \\
V_{m} \sim \frac{g \beta \Delta T}{v}\left(\frac{1}{1+\operatorname{Pr}^{-\frac{1}{2}}}\right)^{2} \kappa t,
\end{gathered}
$$

although in the case of Ref. [41], the temperature difference between the heated plate and the ambient fluid, $\Delta T\left({ }^{\circ} \mathrm{C}\right)$, is constant, while in the current case, $\Delta T$ changes with time as follows:

$$
\Delta T=T_{w}-T_{a}=\Gamma_{w}(t) \Delta_{T}=\Gamma_{w m} \sin (2 \pi f t) \Delta_{T} .
$$

The temperature on the plate is then

$$
T_{w}=\Gamma_{w m} \sin (2 \pi f t) \Delta_{T}+T_{a} \sim \Gamma_{w m} \sin (2 \pi f t) \kappa^{\frac{1}{2}} t^{\frac{1}{2}}+T_{a},
$$

which is the scaling for $T_{w}$ at the start-up stage.

With the time-averaged global Rayleigh number defined for the current time-varying flux case, as shown by (9), the above scaling for $V_{m}$ at the start-up stage, i.e., (13), becomes

$$
V_{m} \sim\left(\frac{\pi}{2}\right) \frac{\kappa^{5 / 2} \operatorname{Ra}}{H^{4}}\left(\frac{1}{1+\operatorname{Pr}^{-\frac{1}{2}}}\right)^{2} \sin (2 \pi f t) t^{3 / 2} .
$$

\section{B. Scalings at the transition time scale $t_{s}$}

At the end of the start-up stage, the heat transferred in through the plate by conduction within the boundary layer will be approximately balanced by the heat carried away by the convection of the flow, and the transitional stage starts. At a height $Y$, by following a similar scaling analysis as that in Ref. [41] for the constant isothermal heating case, this balance leads to the following scaling for the transition time scale $t_{s}$, which represents the ending of the start-up stage and the beginning of the transitional stage for the current case,

$$
t_{s} \sim\left(\frac{2}{\pi}\right)^{2 / 5} \frac{H^{2}(Y / H)^{2 / 5}\left(1+\operatorname{Pr}^{-\frac{1}{2}}\right)^{4 / 5}}{\operatorname{Ra}^{\frac{2}{5}} \kappa\left[\sin \left(2 \pi f t_{s}\right)\right]^{2 / 5}} .
$$

Correspondingly, the scalings for the maximum velocity, thermal boundary-layer thickness, inner viscous boundarylayer thickness, outer viscous boundary-layer thickness, and plate temperature, all at height $Y$ and at the transition time scale $t_{s}$, from (16), (10), (11), (12), and (15), respectively, become

$$
\begin{aligned}
& V_{m, s} \sim\left(\frac{\pi}{2}\right) \frac{\kappa^{5 / 2} \operatorname{Ra}}{H^{4}} \frac{\sin \left(2 \pi f t_{s}\right)}{\left(1+\operatorname{Pr}^{-1 / 2}\right)^{2}} t_{s}^{3 / 2} \\
& \sim\left(\frac{\pi}{2}\right)^{2 / 5} \frac{\kappa \mathrm{Ra}^{2 / 5}}{H}\left(\frac{Y}{H}\right)^{3 / 5} \frac{\left[\sin \left(2 \pi f t_{s}\right)\right]^{2 / 5}}{\left(1+\operatorname{Pr}^{-1 / 2}\right)^{4 / 5}} \\
& \Delta_{T, s} \sim \kappa^{1 / 2} t_{s}^{1 / 2} \\
& \sim\left(\frac{2}{\pi}\right)^{1 / 5} \frac{H}{\operatorname{Ra}^{1 / 5}} \frac{\left(1+\operatorname{Pr}^{-1 / 2}\right)^{2 / 5}}{\left[\sin \left(2 \pi f t_{s}\right)\right]^{1 / 5}}\left(\frac{Y}{H}\right)^{1 / 5} \\
& \Delta_{v i, s} \sim \frac{1}{1+\operatorname{Pr}^{-\frac{1}{2}}} \Delta_{T, s} \\
& \sim\left(\frac{2}{\pi}\right)^{1 / 5} \frac{H}{\operatorname{Ra}^{1 / 5}} \frac{1}{\left(1+\operatorname{Pr}^{-1 / 2}\right)^{3 / 5}\left[\sin \left(2 \pi f t_{s}\right)\right]^{1 / 5}} \\
& \times\left(\frac{Y}{H}\right)^{1 / 5}, \\
& \sim \operatorname{Pr}^{\frac{1}{2}} \Delta_{T, s}{ }^{1 / 5} \frac{H}{\Delta_{v, s}} \frac{\operatorname{Pr}^{1 / 2}\left(1+\operatorname{Pr}^{-1 / 2}\right)^{2 / 5}}{\left[\sin \left(2 \pi f t_{s}\right)\right]^{1 / 5}}\left(\frac{Y}{H}\right)^{1 / 5} \\
& \sim\left(\frac{2}{\pi}\right)^{1 / 5}
\end{aligned}
$$




$$
\begin{aligned}
T_{w, s} \sim & \Gamma_{w m} \sin \left(2 \pi f t_{s}\right) \Delta_{T, s}+T_{a} \\
\sim & \left(\frac{2}{\pi}\right)^{1 / 5} \frac{H}{\mathrm{Ra}^{1 / 5}}\left(\frac{Y}{H}\right)^{1 / 5} \Gamma_{w m}\left(1+\operatorname{Pr}^{-1 / 2}\right)^{2 / 5} \\
& \times\left[\sin \left(2 \pi f t_{s}\right)\right]^{4 / 5}+T_{a}
\end{aligned}
$$

\section{Scalings at the quasi-steady stage}

The mechanisms governing the behavior of the boundary layer during the transitional development stage become quite complicated due to traveling waves caused by the leading edge effect, and it is speculated that no simple scalings can be developed for this stage. Subsequent to the passage of the leading edge waves the boundary layer is at the quasi-steady stage due to the time-varying nature of the flux. At any time instant $t$ at the quasi-steady stage, the convection of heat carried away by the flow again approximately balances the conduction of heat transferred in through the plate. This is similar to the steady state of the constant isothermal heating case and therefore the scaling analysis steps used by Ref. [41] are also applicable for the current case, although appropriate modifications and expansions must be made to take into account of the time-varying nature of the flux, as demonstrated below.

At the height $Y$, the following balance, which was obtained in Ref. [41] from the convection-conduction balance, still holds in the quasi-steady stage of the current case,

$$
\frac{V_{m} \Delta T}{Y} \sim \kappa \frac{\Delta T}{\Delta_{T}^{2}} .
$$

In the vertical momentum equation (5), over $\Delta_{T}$, buoyancy balances viscosity. This leads to the following scaling for $V_{m}$ in region $\mathrm{I}$,

$$
V_{m} \sim \frac{g \beta \Delta T}{v} \Delta_{v i}^{2},
$$

while in region II, the balance leads to the following scaling:

$$
\Delta_{v i} \sim \frac{1}{1+\operatorname{Pr}^{-\frac{1}{2}}} \Delta_{T} .
$$

Both these scalings are the same as those obtained in Ref. [41] for the constant isothermal heating case.

Using (25) and (14), the scaling (24) becomes

$$
V_{m} \sim \frac{g \beta \Gamma_{w}(t) \Delta_{T}^{3}}{v}\left(1+\operatorname{Pr}^{-\frac{1}{2}}\right)^{-2} .
$$

Combining scalings (23) and (26) gives

$$
\frac{\kappa Y}{\Delta_{T}^{2}} \sim \frac{g \beta \Gamma_{w}(t) \Delta_{T}^{3}}{v}\left(1+\operatorname{Pr}^{-\frac{1}{2}}\right)^{-2},
$$

which leads to the following scaling for the thermal boundarylayer thickness at any time $t$ in the quasi-steady state,

$$
\begin{aligned}
\Delta_{T, \mathrm{qs}} & \sim\left[\frac{\kappa \nu Y}{g \beta \Gamma_{w}(t)}\right]^{1 / 5}\left(1+\operatorname{Pr}^{-1 / 2}\right)^{2 / 5} \\
& \sim\left(\frac{2}{\pi}\right)^{1 / 5} \frac{H}{\mathrm{Ra}^{1 / 5}} \frac{\left(1+\operatorname{Pr}^{-1 / 2}\right)^{2 / 5}}{[\sin (2 \pi f t)]^{1 / 5}}\left(\frac{Y}{H}\right)^{1 / 5} .
\end{aligned}
$$

With (28), the scaling (26) becomes

$$
\begin{aligned}
V_{m, \mathrm{qs}} & \sim \frac{g \beta \Gamma_{w}(t) \Delta_{T, \mathrm{qs}}^{3}}{v}\left(1+\operatorname{Pr}^{-\frac{1}{2}}\right)^{-2} \\
& \sim\left(\frac{\pi}{2}\right)^{2 / 5} \frac{\kappa R a^{2 / 5}}{H}\left(\frac{Y}{H}\right)^{3 / 5} \frac{[\sin (2 \pi f t)]^{2 / 5}}{\left(1+\operatorname{Pr}^{-1 / 2}\right)^{4 / 5}},
\end{aligned}
$$

which is the scaling for the maximum vertical velocity within the boundary layer at any time in the quasi-steady state.

It is apparent that the scaling for the plate temperature at height $Y$ at any time in the quasi-steady stage, with (15) and the scaling (28), becomes

$$
\begin{aligned}
T_{w, \mathrm{qs}} \sim & \Gamma_{w m} \sin (2 \pi f t) \Delta_{T, \mathrm{qs}}+T_{a} \\
\sim & \left(\frac{2}{\pi}\right)^{1 / 5} \frac{H}{\mathrm{Ra}^{1 / 5}}\left(\frac{Y}{H}\right)^{1 / 5} \Gamma_{w m}\left(1+\operatorname{Pr}^{-1 / 2}\right)^{2 / 5} \\
& \times[\sin (2 \pi f t)]^{4 / 5}+T_{a} .
\end{aligned}
$$

The scaling for the inner viscous boundary-layer thickness at height $Y$ at any time $t$ in the quasi-steady stage, from (25), is

$$
\begin{aligned}
\Delta_{v i, \mathrm{qs}} \sim & \frac{1}{1+\operatorname{Pr}^{-\frac{1}{2}}} \Delta_{T, \mathrm{qs}} \\
\sim & \left(\frac{2}{\pi}\right)^{1 / 5} \frac{H}{\operatorname{Ra}^{1 / 5}} \frac{1}{\left(1+\operatorname{Pr}^{-1 / 2}\right)^{3 / 5}[\sin (2 \pi f t)]^{1 / 5}} \\
& \times\left(\frac{Y}{H}\right)^{1 / 5} .
\end{aligned}
$$

The scaling for the whole viscous boundary-layer thickness at height $Y$ at any time $t$ in the quasi-steady stage, from (12), is

$$
\begin{aligned}
\Delta_{v, \mathrm{qs}} & \sim \operatorname{Pr}^{\frac{1}{2}} \Delta_{T, \mathrm{qs}} \\
& \sim\left(\frac{2}{\pi}\right)^{1 / 5} \frac{H}{\operatorname{Ra}^{1 / 5}} \frac{\operatorname{Pr}^{1 / 2}\left(1+\operatorname{Pr}^{-1 / 2}\right)^{2 / 5}}{[\sin (2 \pi f t)]^{1 / 5}}\left(\frac{Y}{H}\right)^{1 / 5} .
\end{aligned}
$$

It should be noted that, although the scalings (28)-(32) at the quasi-steady stage are seen to be in the same form as their respective counterparts at the transition time scale $t_{s}$, i.e., (19), (18), (22), (20), and (21), these scalings apply for any time $t$ in the quasi-steady stage, whereas the scalings (18)-(22) are only valid at $t_{s}$.

\section{NONDIMENSIONAL FORMULATION}

To facilitate the numerical validation of the scalings obtained above, the dimensionless forms of the governing equations and the scalings are used. For the natural convection boundary-layer flows considered here, it is natural to choose $H$, the height of the plate, as the characteristic length scale. From (18), it is also natural to choose $V_{0}=\kappa \mathrm{Ra}^{2 / 5} / H(\mathrm{~m} / \mathrm{s})$ as the characteristic velocity scale. Hence, the characteristic time scale for the flows is apparently $\left(H / V_{0}\right)$ and the characteristic pressure scale is $\rho V_{0}^{2}$, where $\rho\left(\mathrm{kg} / \mathrm{m}^{3}\right)$ is the density of fluid. It is also apparent that $\bar{T}_{w}=\bar{\Gamma}_{w} H=2 \Gamma_{w m} H / \pi$ is the appropriate characteristic temperature difference scale.

With these characteristic scales, the governing equations (3)-(6) can be written in the following nondimensional 
forms:

$$
\begin{gathered}
\frac{\partial u}{\partial x}+\frac{\partial v}{\partial y}=0 \\
\frac{\partial u}{\partial \tau}+\frac{\partial(u u)}{\partial x}+\frac{\partial(v u)}{\partial y}=-\frac{\partial p}{\partial x}+\frac{\operatorname{Pr}}{\operatorname{Ra}^{\frac{2}{5}}}\left(\frac{\partial^{2} u}{\partial x^{2}}+\frac{\partial^{2} u}{\partial y^{2}}\right) \\
\frac{\partial v}{\partial \tau}+\frac{\partial(u v)}{\partial x}+\frac{\partial(v v)}{\partial y} \\
=-\frac{\partial p}{\partial y}+\frac{\operatorname{Pr}}{\operatorname{Ra}^{\frac{2}{5}}}\left(\frac{\partial^{2} v}{\partial x^{2}}+\frac{\partial^{2} v}{\partial y^{2}}\right)+\operatorname{PrRa}^{\frac{1}{5}} \theta \\
\frac{\partial \theta}{\partial \tau}+\frac{\partial(u \theta)}{\partial x}+\frac{\partial(v \theta)}{\partial y}=\frac{1}{\operatorname{Ra}^{\frac{2}{5}}}\left(\frac{\partial^{2} \theta}{\partial x^{2}}+\frac{\partial^{2} \theta}{\partial y^{2}}\right)
\end{gathered}
$$

where $x, y, u, v, \tau, p$, and $\theta$ are, respectively the dimensionless forms of $X, Y, U, V, t, P$, and $T$, which are made dimensionless by their respective characteristic scales, i.e.,

$$
\begin{aligned}
& x=\frac{X}{H}, \quad y=\frac{Y}{H}, \quad u=\frac{U}{V_{0}}, \quad v=\frac{V}{V_{0}}, \quad \tau=\frac{t}{\left(H / V_{0}\right)}, \\
& p=\frac{P}{\rho V_{0}^{2}}, \quad \theta=\frac{T-T_{a}}{\bar{T}_{w}} .
\end{aligned}
$$

The origin of the coordinate system is located at the leading edge of the heated plate, at $x=0, y=0$.

\section{A. Dimensionless scalings at the start-up stage}

The scalings at the start-up stage obtained above, i.e., (10), (15), (11), (16), and (12), are made dimensionless as follows:

$$
\begin{gathered}
\delta_{T}=\frac{\Delta_{T}}{H} \sim \frac{\kappa^{1 / 2} t^{1 / 2}}{H} \sim \frac{\kappa^{1 / 2}\left[\left(H / V_{0}\right) \tau\right]^{1 / 2}}{H} \\
\sim\left(\frac{\kappa}{V_{0} H}\right)^{1 / 2} \tau^{1 / 2} \sim \frac{\tau^{1 / 2}}{\operatorname{Ra}^{1 / 5}}, \\
\theta_{w} \sim \frac{T_{w}-T_{a}}{\bar{T}_{w}} \sim \frac{\Gamma_{w m} \sin (2 \pi f t) \Delta_{T}}{(2 / \pi) \Gamma_{w m} H} \sim\left(\frac{\pi}{2}\right) \sin (2 \pi f t) \delta_{T} \\
\sim\left(\frac{\pi}{2}\right) \frac{\sin \left(2 \pi f_{n} \tau\right) \tau^{1 / 2}}{\operatorname{Ra}^{1 / 5}}, \\
\delta_{v i}=\frac{\Delta_{v i}}{H} \sim \frac{1}{1+\operatorname{Pr}^{-1 / 2}} \frac{\kappa^{1 / 2} t^{1 / 2}}{H} \sim \frac{1}{1+\operatorname{Pr}^{-1 / 2}} \delta_{T} \\
\sim \frac{1}{\left(1+\operatorname{Pr}^{-1 / 2}\right) \frac{\tau^{1 / 2}}{\operatorname{Ra}^{1 / 5}},} \\
v_{m}=\frac{V_{m}}{V_{0}} \sim\left(\frac{\pi}{2}\right) \frac{\operatorname{Ra}^{5 / 2}}{H^{4}} \frac{\sin (2 \pi f t)}{\left(1+\operatorname{Pr}^{-1 / 2}\right)^{2}} \frac{t^{3 / 2}}{V_{0}} \\
\sim\left(\frac{\pi}{2}\right) \frac{\operatorname{Ra}^{5 / 2}}{H^{4}} \frac{\sin \left(2 \pi f\left[\tau H / V_{0}\right]\right)}{\left(1+\operatorname{Pr}^{-1 / 2}\right)^{2}} \frac{\left(\tau / V_{0}\right)^{3 / 2}}{V_{0}} \\
\sim\left(\frac{\pi}{2}\right) \frac{\operatorname{Ra}^{5 / 2}}{H^{5 / 2}} \frac{\sin \left(2 \pi f_{n} \tau\right)}{\left(1+\operatorname{Pr}^{-1 / 2}\right)^{2}} \frac{\tau^{3 / 2}}{\left(\kappa / H a^{2 / 5}\right)^{5 / 2}} \\
\sim\left(\frac{\pi}{2}\right) \frac{\sin \left(2 \pi f_{n} \tau\right)}{\left(1+\operatorname{Pr}^{-1 / 2}\right)^{2}} \tau^{3 / 2},
\end{gathered}
$$

$$
\delta_{v}=\frac{\Delta_{v}}{H} \sim \frac{v^{1 / 2} t^{1 / 2}}{H} \sim \operatorname{Pr}^{1 / 2} \delta_{T} \sim \frac{\operatorname{Pr}^{1 / 2} \tau^{1 / 2}}{\mathrm{Ra}^{1 / 5}},
$$

where $f_{n}$ is the dimensionless natural frequency of the timevarying flux applied to the plate which is defined as

$$
f_{n}=\frac{f}{V_{0} / H}=\frac{0.5 / t_{\text {total }}}{V_{0} / H}=\frac{0.5}{\tau_{\text {total }}},
$$

in which $\tau_{\text {total }}=t_{\text {total }} /\left(H / V_{0}\right)$ is the dimensionless total heating time of the time-varying flux applied to the plate.

\section{B. Dimensionless scalings at the dimensionless transition time scale $\tau_{s}$}

The scaling for the transition time scale $t_{s}$, (17), is nondimensionalized as follows:

$$
\tau_{s}=\frac{t_{s}}{\left(H / V_{0}\right)} \sim\left(\frac{2}{\pi}\right)^{2 / 5} \frac{\left(1+\operatorname{Pr}^{-\frac{1}{2}}\right)^{4 / 5} y^{2 / 5}}{\left[\sin \left(2 \pi f_{n} \tau_{s}\right)\right]^{2 / 5}},
$$

and the scalings (18) to (22) are written in the following dimensionless forms:

$$
\begin{gathered}
v_{m, s}=\frac{V_{m, s}}{V_{0}} \sim\left(\frac{\pi}{2}\right)^{2 / 5} \frac{\left[\sin \left(2 \pi f_{n} \tau_{s}\right)\right]^{2 / 5} y^{3 / 5}}{\left(1+\operatorname{Pr}^{-1 / 2}\right)^{4 / 5}}, \\
\delta_{T, s}=\frac{\Delta_{T}}{H} \sim\left(\frac{2}{\pi}\right)^{1 / 5} \frac{\left(1+\operatorname{Pr}^{-1 / 2}\right)^{2 / 5} y^{1 / 5}}{\left[\sin \left(2 \pi f_{n} \tau_{s}\right)\right]^{1 / 5} \mathrm{Ra}^{1 / 5}}, \\
\delta_{v i, s}=\frac{\Delta_{v i, s} \sim}{H} \sim\left(\frac{2}{\pi}\right)^{1 / 5} \frac{y^{1 / 5}}{\left(1+\operatorname{Pr}^{-1 / 2}\right)^{3 / 5}\left[\sin \left(2 \pi f_{n} \tau_{s}\right)\right]^{1 / 5} \mathrm{Ra}^{1 / 5}}, \\
\delta_{v, s}=\frac{\Delta_{v, s} \sim\left(\frac{2}{\pi}\right)^{1 / 5} \frac{\operatorname{Pr}^{1 / 2}\left(1+\operatorname{Pr}^{-1 / 2}\right)^{2 / 5} y^{1 / 5}}{\left[\sin \left(2 \pi f_{n} \tau_{s}\right)\right]^{1 / 5} \mathrm{Ra}^{1 / 5}},}{\theta_{w, s}=}, \\
\quad \frac{T_{w, s}-T_{a}}{\bar{T}_{w}}=\frac{T_{w, s}-T_{a}}{\bar{\Gamma}_{w} H} \sim\left(\frac{\pi}{2}\right)^{4 / 5} \\
\\
\times \frac{\left(1+\operatorname{Pr}^{-1 / 2}\right)^{2 / 5}\left[\sin \left(2 \pi f_{n} \tau_{s}\right)\right]^{4 / 5} y^{1 / 5}}{\operatorname{Ra}^{1 / 5}} .
\end{gathered}
$$

\section{Dimensionless scalings at the quasi-steady stage}

At the quasi-steady stage, the scalings (28)-(32) are made dimensionless as follows:

$$
\begin{aligned}
\delta_{T, \mathrm{qs}}= & \frac{\Delta_{T, \mathrm{qs}}}{H} \sim\left(\frac{2}{\pi}\right)^{1 / 5} \frac{y^{1 / 5}\left(1+\operatorname{Pr}^{-1 / 2}\right)^{2 / 5}}{\mathrm{Ra}^{1 / 5}\left[\sin \left(2 \pi f_{n} \tau\right)\right]^{1 / 5}}, \\
v_{m, \mathrm{qs}}= & \frac{V_{m, \mathrm{qs}}}{V_{0}} \sim\left(\frac{\pi}{2}\right)^{2 / 5} \frac{y^{3 / 5}\left[\sin \left(2 \pi f_{n} \tau\right)\right]^{2 / 5}}{\left(1+\operatorname{Pr}^{-1 / 2}\right)^{4 / 5}}, \\
\theta_{w, \mathrm{qs}}= & \frac{T_{w, \mathrm{qs}}-T_{a}}{\bar{T}_{w}}=\frac{T_{w, \mathrm{qs}}-T_{a}}{\bar{\Gamma}_{w} H} \sim\left(\frac{\pi}{2}\right)^{4 / 5} \\
& \times \frac{y^{1 / 5}\left(1+\operatorname{Pr}^{-1 / 2}\right)^{2 / 5}\left[\sin \left(2 \pi f_{n} \tau\right)\right]^{4 / 5}}{\mathrm{Ra}^{1 / 5}},
\end{aligned}
$$




$$
\begin{aligned}
\delta_{v i, \mathrm{qs}}= & \frac{\Delta_{v i, \mathrm{qs}}}{H} \sim\left(\frac{2}{\pi}\right)^{1 / 5} \\
& \times \frac{y^{1 / 5}}{\mathrm{Ra}^{1 / 5}\left(1+\operatorname{Pr}^{-1 / 2}\right)^{3 / 5}\left[\sin \left(2 \pi f_{n} \tau\right)\right]^{1 / 5}}, \\
\delta_{v, \mathrm{qs}}= & \frac{\Delta_{v, \mathrm{qs}}}{H} \sim\left(\frac{2}{\pi}\right)^{1 / 5} \frac{y^{1 / 5} \operatorname{Pr}^{1 / 2}\left(1+\operatorname{Pr}^{-1 / 2}\right)^{2 / 5}}{\operatorname{Ra}^{1 / 5}\left[\sin \left(2 \pi f_{n} \tau\right)\right]^{1 / 5}} .
\end{aligned}
$$

Scalings (38)-(42) clearly show that at the start-up stage, the boundary-layer development is one-dimensional and independent of $y$ due to the dominance of pure conduction. However, beyond the start-up stage, it becomes two-dimensional and $y$ dependent as the flow is now dominated by convection, as demonstrated by the scalings (45)-(48) at the transition time scale $\tau_{s}$ and (50)-(54) at the quasi-steady stage.

\section{NUMERICAL RESULTS AND DISCUSSIONS}

In this section, the scalings obtained above are validated and analyzed by comparison to a series of DNS results. From the scalings, it is clear that the major parameters that govern the behavior of the unsteady natural convection boundary layer studied in this work are $\mathrm{Ra}, \mathrm{Pr}$, and $f_{n}$. To examine the effects of these three parameters on the scalings obtained above, 10 DNS runs were performed: Four runs are at varying $\mathrm{Ra}(\mathrm{Ra}=$ $10^{6}, 10^{7}, 10^{8}$, and $10^{9}$, respectively) with the fixed $\operatorname{Pr}=7$ and $f_{n}=0.1$ for the $\mathrm{Ra}$ dependence, 5 runs are at varying $\operatorname{Pr}(\operatorname{Pr}=3,7,20,50$, and 100, respectively) with the fixed $\mathrm{Ra}=10^{8}$ and $f_{n}=0.1$ for the Pr dependence, and 3 runs are at varying $f_{n}\left(f_{n}=0.1,0.025\right.$, and 0.01 , respectively) with the fixed $\mathrm{Ra}=10^{8}$ and $\operatorname{Pr}=7$ for the $f_{n}$ dependence.

All simulations were conducted in a dimensionless $2 \times 2$ computational domain with a mesh of $399 \times 396$ nodes. This mesh was chosen after a detailed mesh dependence test which ensures the simulation results obtained with it are mesh independent. The same code used in Refs. [2,18,19,40,41,43-46] was used for these simulations. As the numerical methods, the construction and dependence test of the computational meshes, and the benchmarking of the code against the known theoretical results were well detailed in Refs. [19,40], and [43], and these will not be repeated here.

In the whole stage of the boundary-layer development, it was found that no unique and distinct values can be determined for $\delta_{v}, \delta_{v, s}$, and $\delta_{v, \mathrm{qs}}$ at any height for any DNS runs, due to the flow in region III (i.e., in the outer viscous boundary layer) being driven solely by diffusion of momentum as discussed in Sec. II. Hence, the scalings (42), (48), and (54) cannot be compared with the DNS results. The remaining scalings for $\delta_{T}, \delta_{T, s}, \delta_{T, \mathrm{qs}}, \theta_{w}, \theta_{w, s}, \theta_{w, \mathrm{qs}}, \delta_{v i}, \delta_{v i, s}, \delta_{v i, \mathrm{qs}}, v_{m}, v_{m, s}$, $v_{m, \mathrm{qs}}$, and $\tau_{s}$, i.e., (38)-(41) at the start-up stage, (44)-(49) at the dimensionless transition time $\tau_{s}$, and (50)-(53) at the quasi-steady stage, will be validated by the DNS results in the subsequent sections.

\section{A. Validation of the scalings at the start-up stage}

As shown by the above obtained scalings (38)-(41), the boundary-layer development on the vertical plate at the start-up
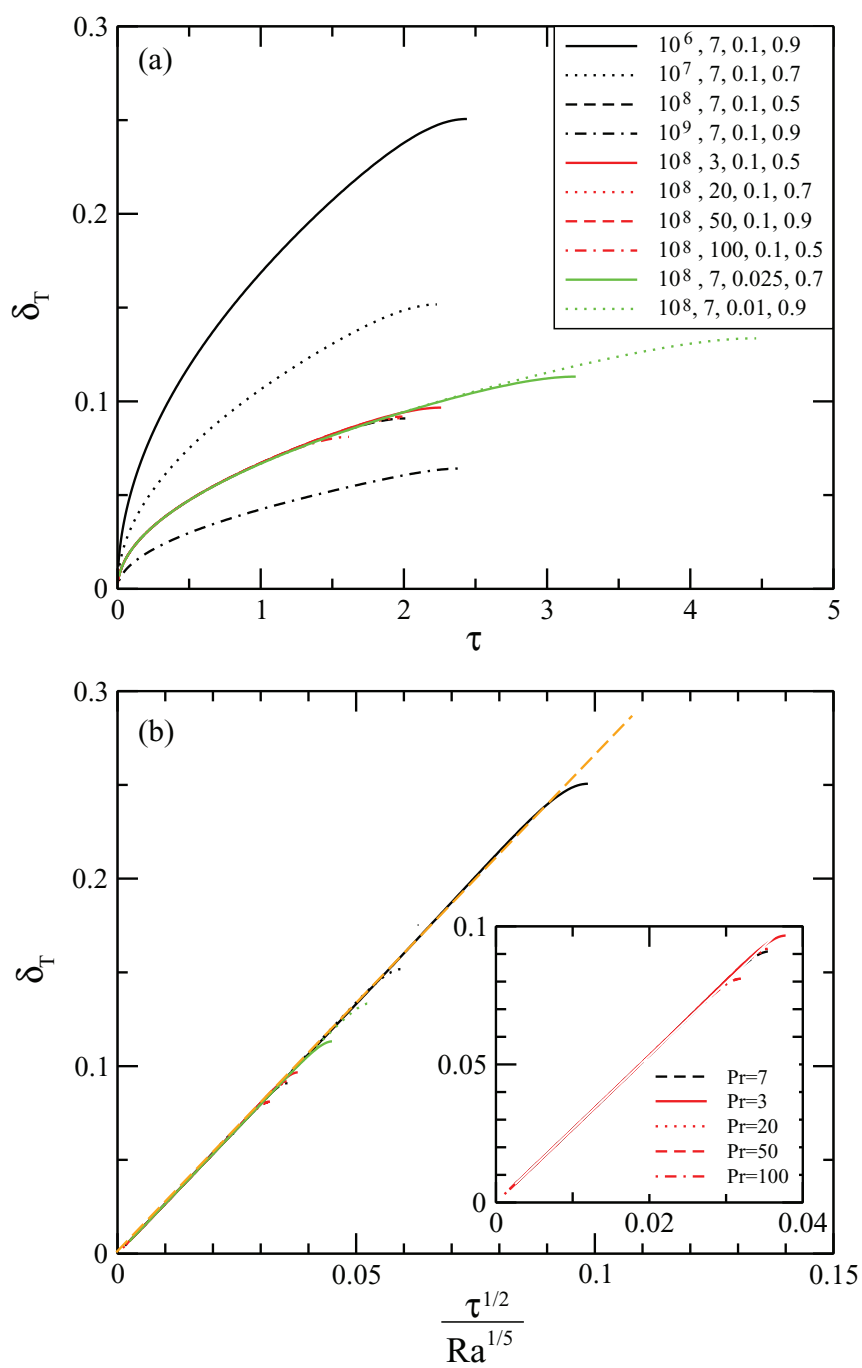

FIG. 2. (Color online) (a) Time series of numerically obtained $\delta_{T}$ and (b) $\delta_{T}$ plotted against $\tau^{1 / 2} / \mathrm{Ra}^{1 / 5}$ at the start-up stage for the 10 DNS runs, each at different heights. The orange dash line in $(b)$ represents the linear correlation obtained by a linear curve-fitting based on the scaled data presented. The notation in the legend in $(a)$, e.g., $10^{8}, 7,0.1,0.5$, represents the DNS run of $\mathrm{Ra}=10^{8}, \mathrm{Pr}=7$, and $f_{n}=0.1$ at the height of $y=0.5$.

stage is one dimensional and $y$ independent. This behavior is clearly confirmed by the DNS results, as described below.

The scaling (38) shows that at the start-up stage the dimensionless thermal boundary-layer thickness, $\delta_{T}$, grows as $\delta_{T} \sim \tau^{1 / 2} / \mathrm{Ra}^{1 / 5}$ and demonstrates no dependence on $\operatorname{Pr}, y$, and $f_{n}$. This is confirmed by the numerical results, as shown in Fig. 2, where the time series of $\delta_{T}$ at the start-up stage and $\delta_{T}$ plotted against $\tau^{1 / 2} / \mathrm{Ra}^{1 / 5}$ are presented for the $10 \mathrm{DNS}$ runs, each at a different height. From Fig. 2(a), it is very evident that the development of $\delta_{T}$ at the start-up stage is independent of Pr, $f_{n}$, and $y$, which supports the scaling (38), as all seven time series at $\mathrm{Ra}=10^{8}$ with different $\operatorname{Pr}, f_{n}$, and $y$ are essentially the same before they approach their respective ends of the start-up stages. The remaining three time series at different $\mathrm{Ra}$ clearly show that the development of $\delta_{T}$ at the start-up stage is $\mathrm{Ra}$ dependent, which is apparently in agreement with the 

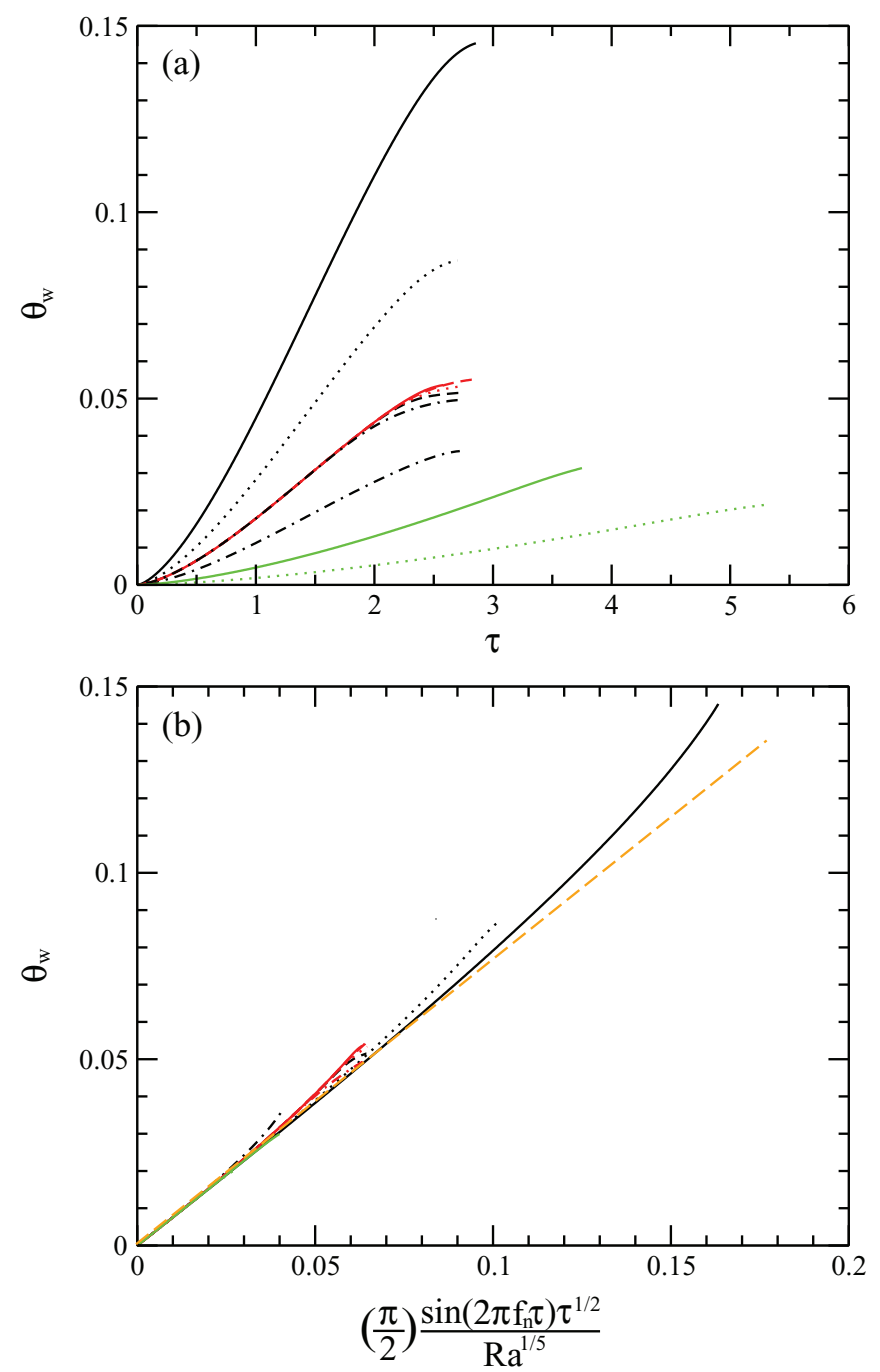

FIG. 3. (Color online) (a) Time series of numerically obtained $\theta_{w}$ and (b) $\theta_{w}$ plotted against $(\pi / 2) \sin \left(2 \pi f_{n} \tau\right) \tau^{1 / 2} / \mathrm{Ra}^{1 / 5}$ at the start-up stage for the 10 DNS runs, each at different heights. The orange dash line in (b) represents the linear correlation obtained by a linear curve-fitting based on the scaled data presented. The legend and its notations are the same as those in Fig. 2.

scaling (38). The results presented in Fig. 2(b) further show that the relation $\delta_{T} \sim \tau^{1 / 2} / \mathrm{Ra}^{1 / 5}$ collapses all 10 time series at the start-up stage onto a single straight line represented by

$$
\delta_{T}=2.676 \tau^{1 / 2} / \mathrm{Ra}^{1 / 5},
$$

which clearly confirms the scaling (38).

At the start-up stage, the dimensionless wall temperature, $\theta_{w}$, is expected to grow following $\theta_{w} \sim$ $(\pi / 2) \sin \left(2 \pi f_{n} \tau\right) \tau^{1 / 2} / \mathrm{Ra}^{1 / 5}$, as predicted by the scaling (39), and has no dependence on $\operatorname{Pr}$ and $y$. The DNS results validate this scaling, as demonstrated in Fig. 3, where the time series of $\theta_{w}$ at the start-up stage and $\theta_{w}$ plotted against $(\pi / 2) \sin \left(2 \pi f_{n} \tau\right) \tau^{1 / 2} / \mathrm{Ra}^{1 / 5}$ are presented for the 10 DNS runs, each at a different height. Figure 3(a) clearly shows that the development of $\theta_{w}$ at the start-up stage is independent of Pr and $y$, as all five time series at $\mathrm{Ra}=10^{8}$ and $f_{n}=0.1$ with different $\operatorname{Pr}$ and $y$ are basically the same before they appraoch their respective $\tau_{s}$. The four time series at different $\mathrm{Ra}$ and the three time series at different $f_{n}$ demonstrate that the development of $\theta_{w}$ at the start-up stage is $\mathrm{Ra}$ and $f_{n}$ dependent. These observations are in agreement with the scaling (39). This scaling is more evidently validated by the results presented in Fig. 3(b), which show that the relation $\theta_{w} \sim(\pi / 2) \sin \left(2 \pi f_{n} \tau\right) \tau^{1 / 2} / \mathrm{Ra}^{1 / 5}$ collapses all 10 time series at the start-up stage onto the same straight line represented by

$$
\theta_{w}=0.767(\pi / 2) \sin \left(2 \pi f_{n} \tau\right) \tau^{1 / 2} / \mathrm{Ra}^{1 / 5},
$$

confirming that the scaling (39) is the correct scaling for $\theta_{w}$ at the start-up stage.

From the scaling (40), it is seen that at the start-up stage the dimensionless inner viscous boundary-layer thickness, $\delta_{v i}$, grows like $\delta_{v i} \sim \tau^{1 / 2} /\left(1+\operatorname{Pr}^{-1 / 2}\right) / \mathrm{Ra}^{1 / 5}$ and does not depend on $f_{n}$ or $y$. The raw data presented in Fig. 4(a), where the time series of $\delta_{v i}$ at the start-up stage are presented for the 10 DNS runs, each at a different height, show that the development of
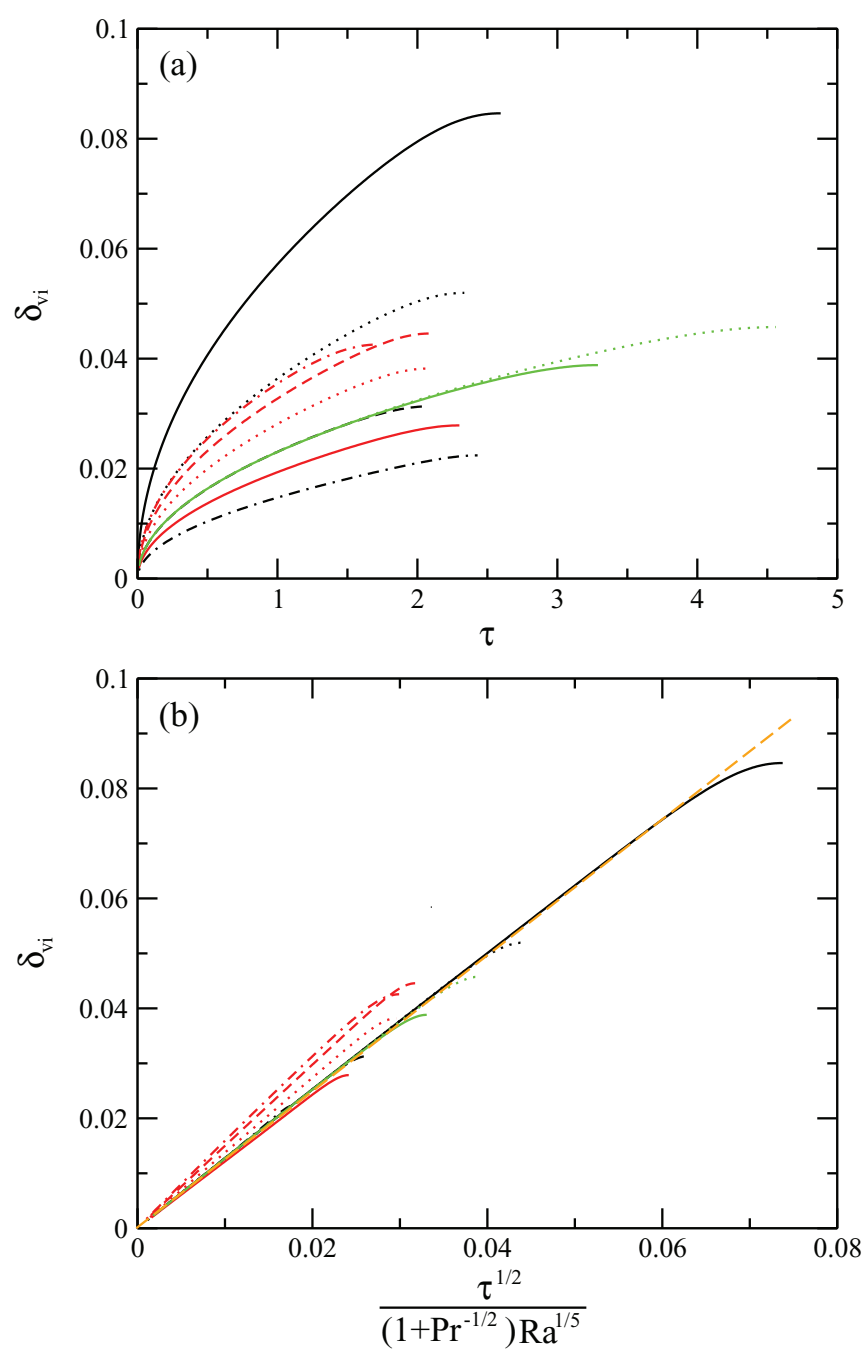

FIG. 4. (Color online) (a) Time series of numerically obtained $\delta_{v i}$ and (b) $\delta_{v i}$ plotted against $\tau^{1 / 2} /\left(1+\mathrm{Pr}^{-1 / 2}\right) / \mathrm{Ra}^{1 / 5}$ at the start-up stage for the 10 DNS runs, each at different heights. The orange dash line in (b) represents the linear correlation obtained by a linear curve-fitting based on the scaled data presented. The legend and its notations are the same as those in Fig. 2. 
$\delta_{v i}$ at the start-up stage is indeed independent of $f_{n}$ and $y$, as all three time series at $\mathrm{Ra}=10^{8}$ and $\operatorname{Pr}=7$ with different $f_{n}$ and $y$ are in fact the same before their respective start-up stages come to the end. The remaining time series at different $\mathrm{Ra}$ and Pr clearly show that the development of $\delta_{v i}$ at the start-up stage is $\mathrm{Ra}$ and Pr dependent, which is again in agreement with the scaling (40). Figure 4(b), which plots the time series of $\delta_{v i}$ at the start-up stage against $\tau^{1 / 2} / \mathrm{Ra}^{1 / 5} /\left(1+\operatorname{Pr}^{-1 / 2}\right)$ for the 10 DNS runs, each at a different height, shows that the relation $\delta_{v i} \sim \tau^{1 / 2} / \mathrm{Ra}^{1 / 5} /\left(1+\operatorname{Pr}^{-1 / 2}\right)$ collapses all time series, except those with $\operatorname{Pr}>7$, onto the same straight line represented by

$$
\delta_{v i}=1.251 \tau^{1 / 2} /\left(1+\operatorname{Pr}^{-1 / 2}\right) / \mathrm{Ra}^{1 / 5},
$$

which confirms the dependence of the scaling (40) on $\mathrm{Ra}$ and $f_{n}$ and its independence of $y$. The noticeable deviations of the three time series with Pr larger than 7, although each of which is a straight line after scaling which confirms the $\tau^{1 / 2}$ dependence for each $\operatorname{Pr}$ value, indicate that in addition to the Pr dependence predicted by the scaling (40), i.e., $\delta_{v i} \sim$ $\left(1+\operatorname{Pr}^{-1 / 2}\right), \delta_{v i}$ must have a further, although relatively weak, dependence on Pr that the current scaling analysis, obtained using the simple three-region structure for the unsteady natural convection boundary layer adjacent to the vertical plate for Pr $>1$ fluids, is unable to predict. A similar behavior was also observed for $\delta_{v i}$ for the case of the unsteady natural convection boundary layer adjacent to the vertical plate with constant isothermal heating, as discussed by Lin and Armfield [2]. As argued by Carey and Mollendorf [10] for the uniform heat flux case, large disagreement with the experimental results at very high Pr can be attributed to the non-boundary-layer nature of the velocity field. Hence, it is reasonable to speculate that the deviations observed above for $\delta_{v i}$ are caused by some approximations made in the scaling analysis in Sec. II. In particular, the approximations $(\partial V / \partial X)_{\Delta T} \sim V_{m} /\left(\Delta_{v}-\Delta_{v i}\right)$ and $\int_{\Delta_{v i}}^{\Delta_{T}} \Delta T d x \sim \Delta T\left(\Delta_{T}-\Delta_{v i}\right)$ used in (10) of Ref. [41] are believed to be the major causes for the deviations from the scalings for $\Delta_{v i}$ and $V_{m}$ (as shown below). However, a further study will be needed to find better approximations, which is beyond the scope of the current investigation.

The dimensionless maximum vertical velocity within the viscous boundary layer, $v_{m}$, at the start-up stage, is expected to grow like $v_{m} \sim(\pi / 2) \sin \left(2 \pi f_{n} \tau\right) \tau^{3 / 2}\left(1+\operatorname{Pr}^{-1 / 2}\right)^{2}$, as shown by the scaling (41), and is expected to be independent of Ra and $y$. The DNS results also validate this scaling, as demonstrated in Fig. 5, where the time series of $v_{m}$ at the start-up stage and $v_{m}$ plotted against $(\pi / 2) \sin \left(2 \pi f_{n} \tau\right) \tau^{3 / 2} /\left(1+\operatorname{Pr}^{-1 / 2}\right)^{2}$ are presented for the 10 DNS runs, each at a different height. From Fig. 5(a), it is seen that the development of $v_{m}$ at the start-up stage is clearly independent of $\mathrm{Ra}$ and $y$, as all four time series at $\operatorname{Pr}=7$ and $f_{n}=0.1$ with different $\mathrm{Ra}$ and $y$ fall onto the same curve before they attain the ends of their respective start-up stages. The distinctively different curves of the remaining time series with different $\operatorname{Pr}$ and $f_{n}$ clearly confirm that the development of $v_{m}$ at the start-up stage is $\operatorname{Pr}$ and $f_{n}$ dependent. Figure 5(b) shows that the relation $v_{m} \sim(\pi / 2) \sin \left(2 \pi f_{n} \tau\right) \tau^{3 / 2} /\left(1+\operatorname{Pr}^{-1 / 2}\right)^{2}$ collapses all time series, except the two time series with smaller $f_{n}$ values (i.e., $f_{n}=0.025$ and $\left.f_{n}=0.01\right)$, roughly onto a single straight line
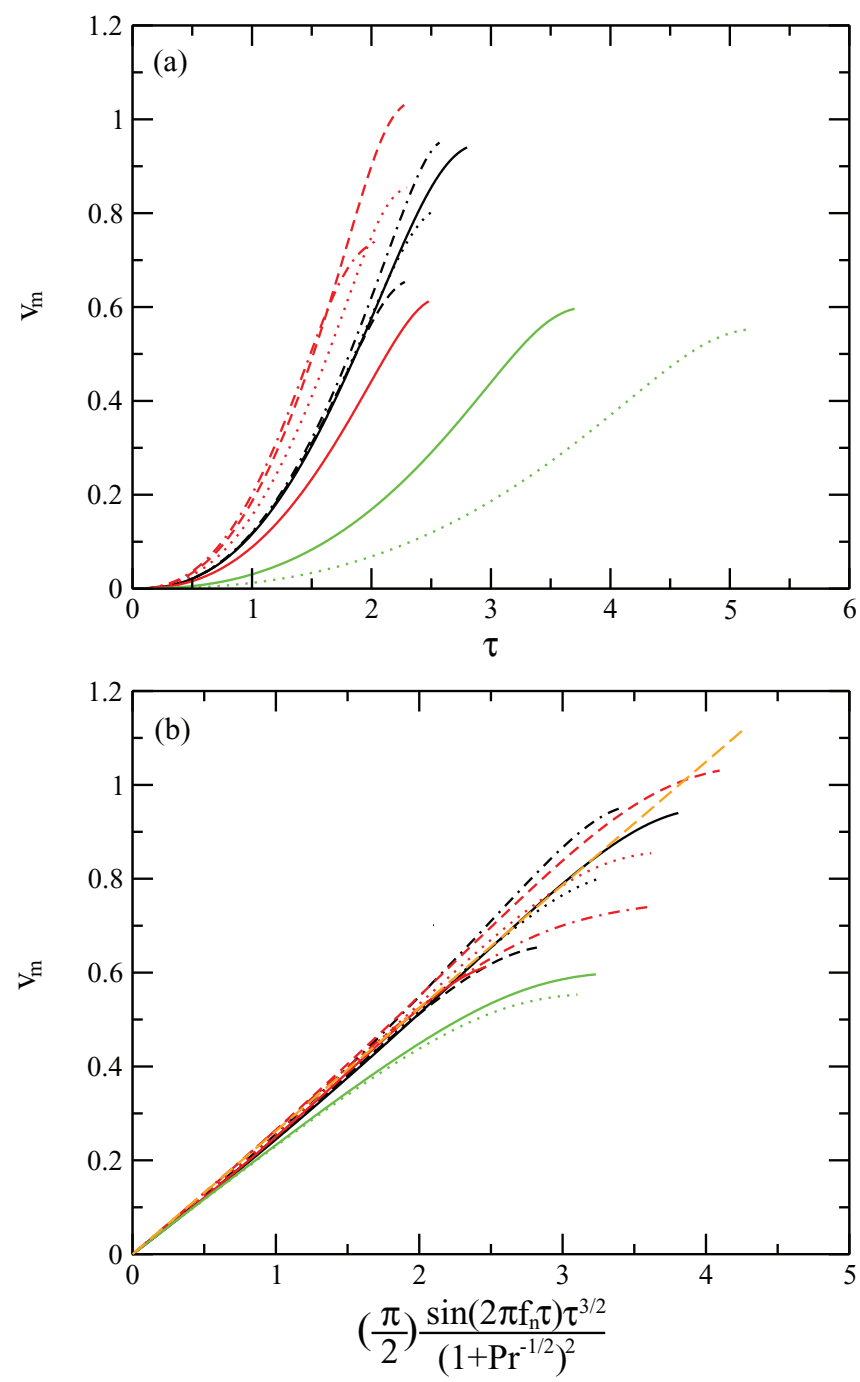

FIG. 5. (Color online) (a) Time series of numerically obtained $v_{m}$ and (b) $v_{m}$ plotted against $(\pi / 2) \sin \left(2 \pi f_{n} \tau\right) \tau^{3 / 2} /\left(1+\operatorname{Pr}^{-1 / 2}\right)^{2}$ at the start-up stage for the 10 DNS runs, each at different heights. The orange dash line in (b) represents the linear correlation obtained by a linear curve-fitting based on the scaled data presented. The legend and its notations are the same as those in Fig. 2.

represented by

$$
v_{m}=0.261(\pi / 2) \sin \left(2 \pi f_{n} \tau\right) \tau^{3 / 2}\left(1+\operatorname{Pr}^{-1 / 2}\right)^{2},
$$

confirming the dependence of the scaling (41) on Pr and its independence of $\mathrm{Ra}$ and $y$. The two time series with $f_{n}=$ 0.025 and 0.01 also scale to straight lines, confirming the time dependence for each $f_{n}$ value. The deviations of the scaled results at these $f_{n}$ indicate that in addition to the predicted $v_{m} \sim \sin \left(2 \pi f_{n} \tau\right) \tau^{3 / 2}$ relation, $v_{m}$ must have a further, weak, dependence on $f_{n}$, not captured by the current analysis, similar to $\delta_{v i}$ as discussed above.

\section{B. Validation of the scalings at the transition time scale $\tau_{s}$}

At the transition time scale $\tau_{s}$, the scalings (45)-(49) show that the boundary layer becomes two dimensional and $y$ dependent as the flow is dominated by convection, as stated 


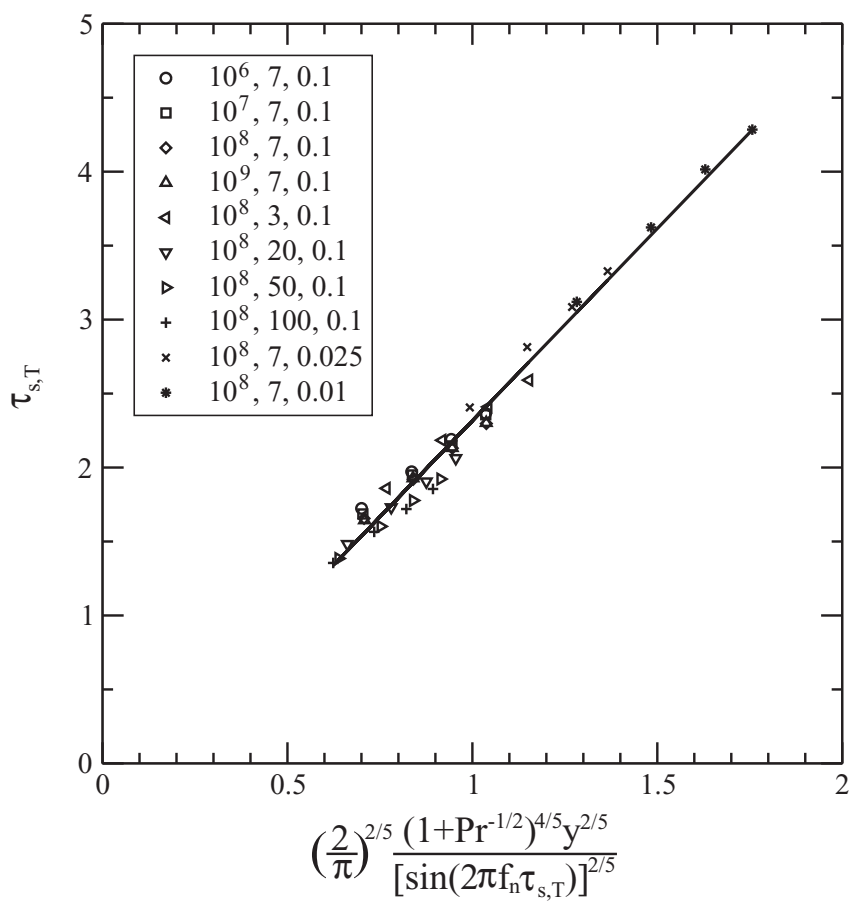

FIG. 6. Numerically obtained $\tau_{s, T}$ plotted against $(2 / \pi)^{2 / 5}(1+$ $\left.\operatorname{Pr}^{-1 / 2}\right)^{4 / 5} y^{2 / 5} /\left[\sin \left(2 \pi f_{n} \tau_{s, T}\right)\right]^{2 / 5}$ for the 10 DNS runs. The solid line represents the linear correlation obtained by a linear curve-fitting based on the scaled data presented. The notation in the legend, e.g., $10^{8}, 7,0.1$, represents the DNS run of $\mathrm{Ra}=10^{8}, \mathrm{Pr}=7$, and $f_{n}=0.1$.

in Sec. IIIC. These scalings are also confirmed by the DNS results, as detailed below.

Although the DNS results demonstrate that the start-up stages terminate at different time instants for $\delta_{T}, \theta_{w}, \delta_{v i}$, and $v_{m}$ (i.e., the transition time $\tau_{s}$ has different valus for these parameters); nevertheless, all these parameters should have, from the scaling perspective, the same time scale $\tau_{s}$ to quantify this critical, termination time instant of the start-up stage. Hence, for convenience and simplicity the time scale representing the termination instant of the start-up stage for $\delta_{T}$, i.e., $\tau_{s, T}$, which is also the transition time scale for $\delta_{T}$, is selected as the time scale to quantify the termination time of the start-up stage for all these parameters, $\delta_{T}, \theta_{w}, \delta_{v i}$, and $v_{m}$.

The scaling for $\tau_{s, T}$ is (44), which states that $\tau_{s} \sim$ $(2 / \pi)^{2 / 5}\left(1+\operatorname{Pr}^{-\frac{1}{2}}\right)^{4 / 5} y^{2 / 5} /\left[\sin \left(2 \pi f_{n} \tau_{s}\right)\right]^{2 / 5}$, indicating that $\tau_{s}$ depends on Pr, $f_{n}$, and $y$ but is independent of Ra. This is validated by the DNS results as shown in Fig. 6 where the numerically obtained $\tau_{s, T}$ is plotted against $(2 / \pi)^{2 / 5}(1+$ $\left.\operatorname{Pr}^{-1 / 2}\right)^{4 / 5} y^{2 / 5} /\left[\sin \left(2 \pi f_{n} \tau_{s, T}\right)\right]^{2 / 5}$ for the 10 DNS runs. For each run, the $\tau_{s, T}$ data at four different heights (i.e., at $y=0.3$, $0.5,0.7$, and 0.9 , respectively) are included in the figure. It is observed that the scaled data roughly fall onto a straight line represented by

$$
\tau_{s, T}=-0.282+2.598\left(\frac{2}{\pi}\right)^{2 / 5} \frac{\left(1+\operatorname{Pr}^{-\frac{1}{2}}\right)^{4 / 5} y^{2 / 5}}{\left[\sin \left(2 \pi f_{n} \tau_{s}\right)\right]^{2 / 5}},
$$

which was obtained by a linear curve fitting. The regression coefficient of this best-fit line is 0.9907 , indicating that it is a reasonably good correlation and, hence, the scaling (44)

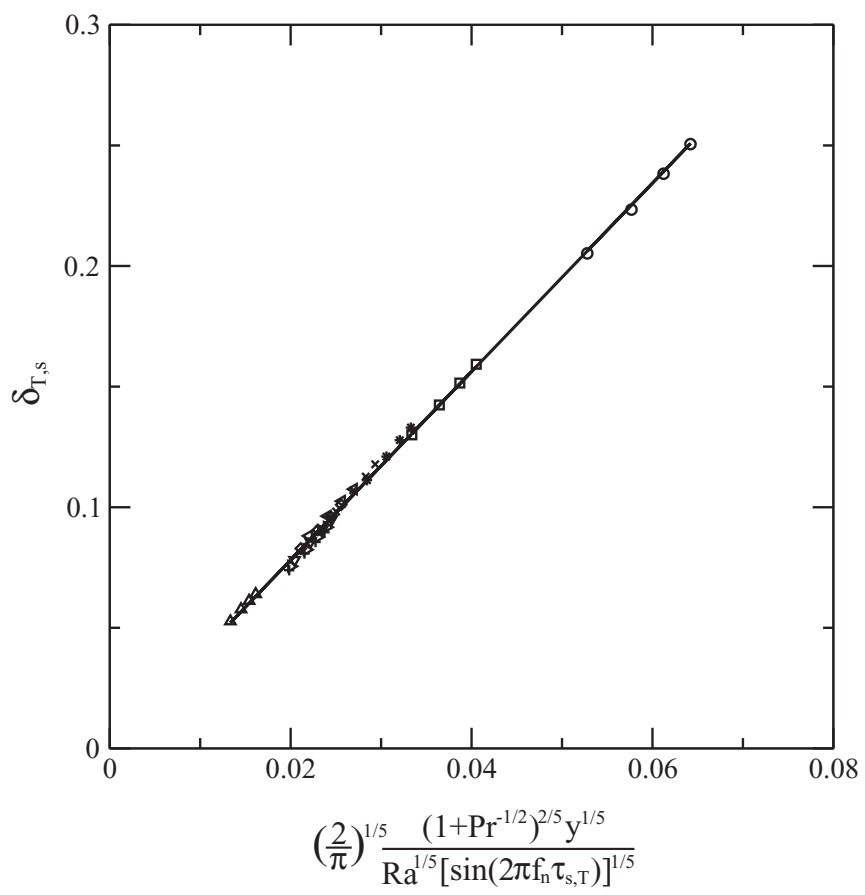

FIG. 7. Numerically obtained $\delta_{T, s}$ plotted against $(2 / \pi)^{1 / 5}(1+$ $\left.\operatorname{Pr}^{-1 / 2}\right)^{2 / 5} y^{1 / 5} / \mathrm{Ra}^{1 / 5} /\left[\sin \left(2 \pi f_{n} \tau_{s, T}\right)\right]^{1 / 5}$ for the 10 DNS runs. The solid line represents the linear correlation obtained by a linear curve-fitting based on the scaled data presented. The legend and its notations are the same as those in Fig. 6.

for $\tau_{s, T}$ at the transition time scale is confirmed by the DNS results. It was also found that close to the leading-edge of the plate (i.e., close to $y=0$ ) noticeable deviations of the DNS results from the prediction by the scaling are observed due to the fact that in this region the boundary-layer assumption (i.e., $\delta_{T} \ll y, \delta_{v i} \ll y$, and $\left.\delta_{v} \ll y\right)$ is not sufficiently satisfied. The deviation observed for the higher-Pr results indicates a further Pr dependency not fully captured by the scaling, as discussed above.

At the transition time scale $\tau_{s, T}$, the scaling for the dimensionless thermal boundary-layer thickness, $\delta_{T, s}$, is (46), which shows that $\delta_{T, s}$ depends on all three governing parameters $\mathrm{Ra}$, Pr, and $f_{n}$, as well as being $y$ dependent. This scaling is well validated by the DNS results, as shown in Fig. 7 where the numerically obtained $\delta_{T, s}$ is plotted against $(2 / \pi)^{1 / 5}(1+$ $\left.\operatorname{Pr}^{-1 / 2}\right)^{2 / 5} y^{1 / 5} / \mathrm{Ra}^{1 / 5} /\left[\sin \left(2 \pi f_{n} \tau_{s, T}\right)\right]^{1 / 5}$ for all 10 runs, with each run containing four sets of data at the heights $y=0.3$, $0.5,0.7$, and 0.9. The figure demonstrates that the scaled data fall very well onto the same straight line quantified by the following best-fit line,

$$
\delta_{T, s}=3.906\left(\frac{2}{\pi}\right)^{1 / 5} \frac{\left(1+\operatorname{Pr}^{-1 / 2}\right)^{2 / 5} y^{1 / 5}}{\left[\sin \left(2 \pi f_{n} \tau_{s}\right)\right]^{1 / 5} \mathrm{Ra}^{1 / 5}},
$$

with a regression coefficient of 0.9992 , indicating that the DNS results are in excellent agreement with the scaling (46). The $\delta_{T, s} \sim \mathrm{Ra}^{1 / 5}$ obtained above is apparently in agreement with the theoretical prediction of Ref. [13], although this theoretical prediction was obtained for the case with constant prescribed fluxes of heat or mass at the vertical walls. 


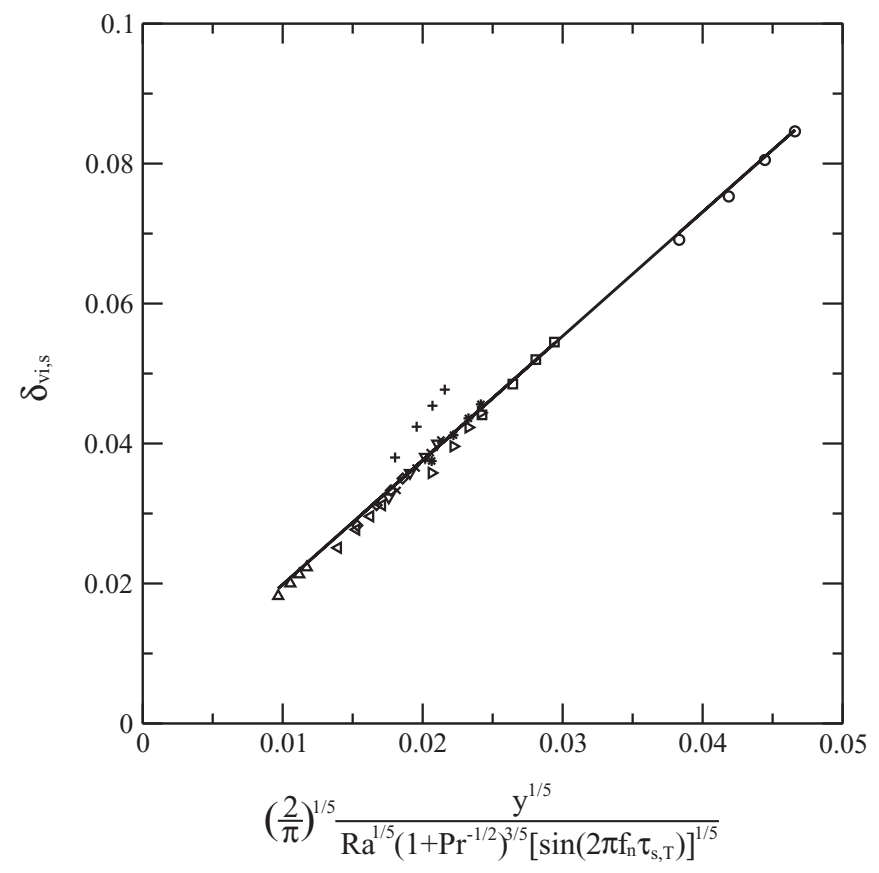

FIG. 8. Numerically obtained $\delta_{v i, s}$ plotted against $(2 / \pi)^{1 / 5} y^{1 / 5} /$ $\mathrm{Ra}^{1 / 5} /\left(1+\operatorname{Pr}^{-1 / 2}\right)^{3 / 5} /\left[\sin \left(2 \pi f_{n} \tau_{s, T}\right)\right]^{1 / 5}$ for the 10 DNS runs. The solid line represents the linear correlation obtained by a linear curvefitting based on the scaled data presented. The legend and its notations are the same as those in Fig. 6.

Similarly, (47), which is the scaling for the dimensionless inner viscous boundary-layer thickness at transition time scale, $\delta_{v i, s}$, demonstrates that $\delta_{v i, s}$ also depends on Ra, $\operatorname{Pr}, f_{n}$, and $y$. This is again validated by the DNS results, as shown in Fig. 8 , in which the numerically obtained $\delta_{v i, s}$ is plotted against $(2 / \pi)^{1 / 5} y^{1 / 5} / \mathrm{Ra}^{1 / 5} /\left(1+\operatorname{Pr}^{-1 / 2}\right)^{3 / 5} /\left[\sin \left(2 \pi f_{n} \tau_{s, T}\right)\right]^{1 / 5}$ for all 10 runs. The figure demonstrates that all data, except the data for the case of $\mathrm{Ra}=10^{8}, \operatorname{Pr}=100$, and $f_{n}=0.1$, fall well onto the same straight line quantified by

$$
\begin{aligned}
\delta_{v i, s}= & 0.002+1.775\left(\frac{2}{\pi}\right)^{1 / 5} \\
& \times \frac{y^{1 / 5}}{\left(1+\operatorname{Pr}^{-1 / 2}\right)^{3 / 5}\left[\sin \left(2 \pi f_{n} \tau_{s}\right)\right]^{1 / 5} \mathrm{Ra}^{1 / 5}},
\end{aligned}
$$

which was obtained by a linear curve-fitting. The regression coefficient of this best-fit line is 0.9899 , indicating the DNS results are also in good agreement with the scaling (47). The noticeable deviations produced by the $\operatorname{Pr}=100$ case is due to the same reason as discussed above for the scaling for $\delta_{v i}$ at the start-up stage, i.e., the scaling must have a further, although weak, dependence on $\mathrm{Pr}$, in addition to that predicted by the scaling (47), which the current scaling analysis using the simple three-region structure is unable to predict.

The scaling for the dimensionless maximum vertical velocity within the boundary layer at the transition time scale, $v_{m, s}$, is (45), which shows that $v_{m, s} \sim$ $(\pi / 2)^{2 / 5}\left[\sin \left(2 \pi f_{n} \tau_{s}\right)\right]^{2 / 5} y^{3 / 5} /\left(1+\operatorname{Pr}^{-1 / 2}\right)^{4 / 5}$, indicating that $v_{m, s}$ depends on $\operatorname{Pr}, f_{n}$, and $y$ but not on Ra. This scaling is also confirmed by the DNS results as demonstrated in

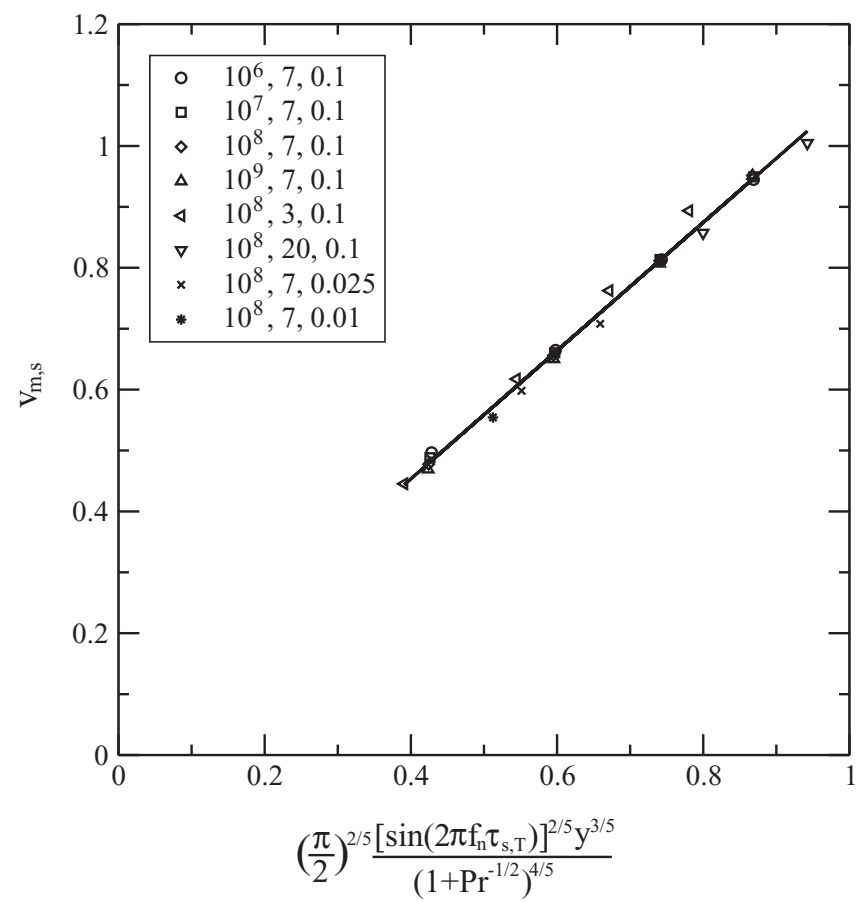

FIG. 9. Numerically obtained $v_{m, s}$ plotted against $(\pi / 2)^{2 / 5}$ $\left[\sin \left(2 \pi f_{n} \tau_{s, T}\right)\right]^{2 / 5} y^{3 / 5} /\left(1+\operatorname{Pr}^{-1 / 2}\right)^{4 / 5}$ for eight DNS runs (No unique and distinct values for $v_{m, s}$ can be identified for the DNS runs with $\operatorname{Pr}=50$ and $\operatorname{Pr}=100$ ). The solid line represents the linear correlation obtained by a linear curve-fitting based on the scaled data presented. The notations in the legend are the same as those in Fig. 6.

Fig. 9, where the numerically obtained $v_{m, s}$ is plotted against $(\pi / 2)^{2 / 5}\left[\sin \left(2 \pi f_{n} \tau_{s}\right)\right]^{2 / 5} y^{3 / 5} /\left(1+\operatorname{Pr}^{-1 / 2}\right)^{4 / 5}$ for eight DNS runs, with each run including data at several heights. The figure does not contain the data for the high-Pr runs (i.e., $\operatorname{Pr}=50$ and $\operatorname{Pr}=100$ runs) as no unique and distinct values can be identified from the DNS results for $v_{m, s}$ at any height for these two cases. It is observed that all data presented in Fig. 9 fall reasonably well onto a straight line represented by

$$
v_{m, s}=0.032+1.053\left(\frac{\pi}{2}\right)^{2 / 5} \frac{\left[\sin \left(2 \pi f_{n} \tau_{s}\right)\right]^{2 / 5} y^{3 / 5}}{\left(1+\operatorname{Pr}^{-1 / 2}\right)^{4 / 5}},
$$

which was obtained by a linear curve fitting. The regression coefficient of this best-fit line is 0.9971 , confirming that it is a reasonably good correlation and, hence, the scaling (45) for $v_{m, s}$ at the transition time scale is confirmed by the DNS results.

At the transition time scale, similarly to $\delta_{v}$ and $\delta_{v, s}$, no unique and distinct values can be determined for $\theta_{w, s}$ at any height for any DNS runs, and, hence, the scaling (49) cannot be validated directly by the DNS results. However, scaled horizontal temperature profiles, presented in Sec. IV D, will provide validation for this scaling.

\section{Validation of the scalings at the quasi-steady stage}

The scalings at the quasi-steady stage, i.e., (50)-(53), are also found generally to be in good agreement with the DNS results. 


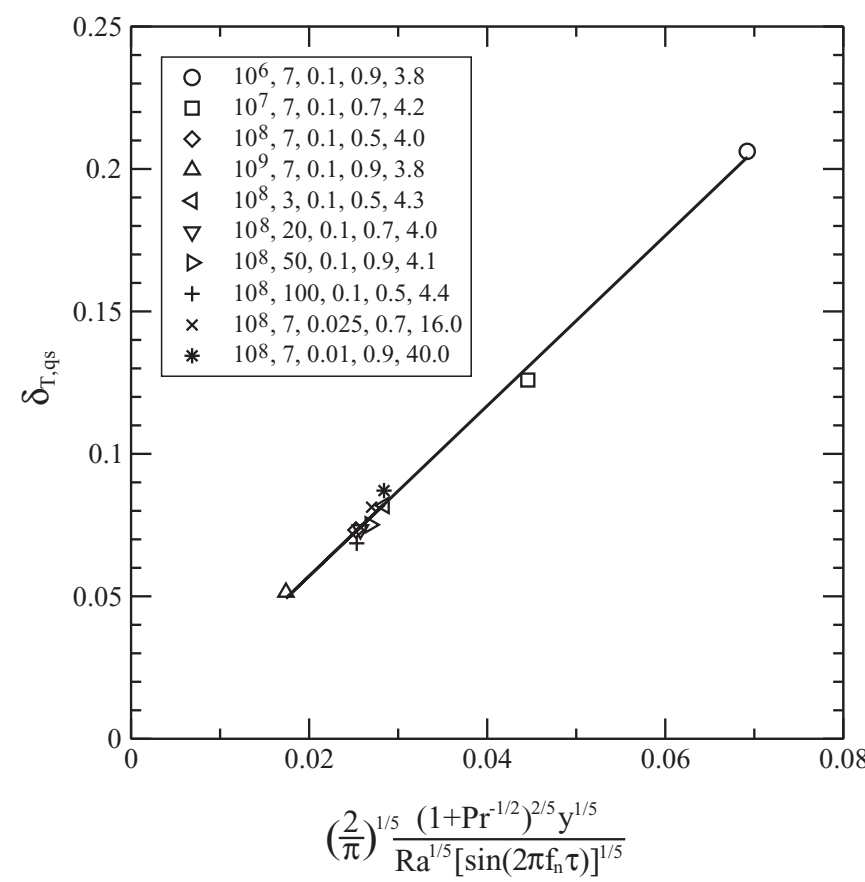

FIG. 10. Numerically obtained $\delta_{T, \text { qs }}$ plotted against $(2 / \pi)^{1 / 5}(1+$ $\left.\operatorname{Pr}^{-1 / 2}\right)^{2 / 5} y^{1 / 5} / \mathrm{Ra}^{1 / 5} /\left[\sin \left(2 \pi f_{n} \tau\right)\right]^{1 / 5}$ for the 10 DNS runs. The solid line represents the linear correlation obtained by a linear curve-fitting based on the scaled data presented. The notation in the legend, e.g., $10^{8}, 7,0.1,0.5,4.0$ represents the DNS run of $\mathrm{Ra}=10^{8}, \mathrm{Pr}=7$, and $f_{n}=0.1$ at the height of $y=0.5$ and at the time instant $\tau=4.0$.

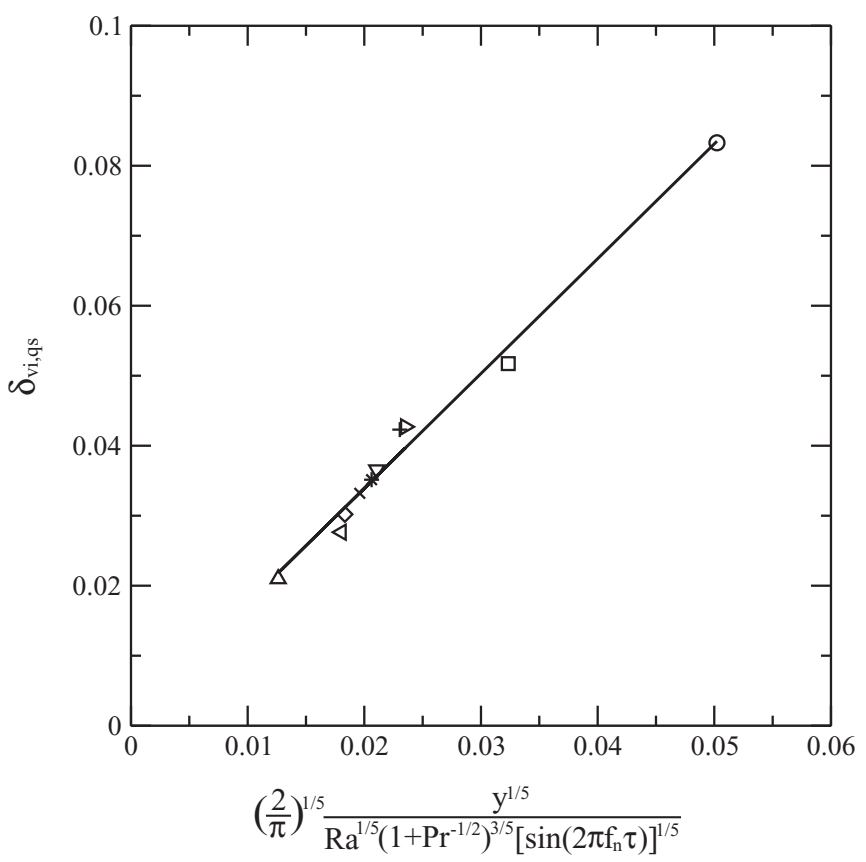

FIG. 11. Numerically obtained $\delta_{v i \text {,qs }}$ plotted against $(2 / \pi)^{1 / 5} y^{1 / 5} /$ $\mathrm{Ra}^{1 / 5} /\left(1+\operatorname{Pr}^{-1 / 2}\right)^{3 / 5} /\left[\sin \left(2 \pi f_{n} \tau\right)\right]^{1 / 5}$ for the 10 DNS runs. The solid line represents the linear correlation obtained by a linear curve-fitting based on the scaled data presented. The legend and its notations are the same as those in Fig. 10.
The scaling for the dimensionless thermal boundary-layer thickness at the quasi-steady stage, $\delta_{T, \mathrm{qs}}$, is (50), which shows that $\delta_{T, \mathrm{qs}}$ depends not only on all three governing parameters $\mathrm{Ra}, \operatorname{Pr}$, and $f_{n}$ but is also $y$ dependent. Figure 10 presents the numerically obtained $\delta_{T \text {,qs }}$ plotted against $(2 / \pi)^{1 / 5}(1+$ $\left.\operatorname{Pr}^{-1 / 2}\right)^{2 / 5} y^{1 / 5} / \mathrm{Ra}^{1 / 5} /\left[\sin \left(2 \pi f_{n} \tau\right)\right]^{1 / 5}$ for all 10 runs, with each run at one of three heights of $y=0.5,0.7$, and 0.9 and at a range of $\tau$, all in the predicted quasi-steady stage. It is seen that the scaling (50) collapses all data well onto the same straight line quantified by

$$
\delta_{T, \mathrm{qs}}=-0.0027+2.9879\left(\frac{2}{\pi}\right)^{1 / 5} \frac{\left(1+\operatorname{Pr}^{-1 / 2}\right)^{2 / 5} y^{1 / 5}}{\left[\sin \left(2 \pi f_{n} \tau\right)\right]^{1 / 5} \mathrm{Ra}^{1 / 5}}
$$

which was obtained by a linear curve fitting, with a regression coefficient of 0.9974 .

Similarly, (53), which is the scaling for the dimensionless inner viscous boundary layer-thickness at the quasi-steady stage, $\delta_{v i, \mathrm{qs}}$, shows that $\delta_{v i, \mathrm{qs}}$ also depends on $\mathrm{Ra}, \operatorname{Pr}, f_{n}$, and $y$, which is again validated by the DNS results, as demonstrated in Fig. 11. This figure presents the numerically obtained $\delta_{v i, \mathrm{qs}}$ plotted against $(2 / \pi)^{1 / 5} y^{1 / 5} / \mathrm{Ra}^{1 / 5} /(1+$ $\left.\operatorname{Pr}^{-1 / 2}\right)^{3 / 5} /\left[\sin \left(2 \pi f_{n} \tau\right)\right]^{1 / 5}$ for the same 10 cases as those for Fig. 10. It is shown that the scaling (53) collapes all data well onto the same straight line quantified by

$$
\begin{aligned}
\delta_{v i, \mathrm{qs}}= & 0.0011+1.6402\left(\frac{2}{\pi}\right)^{1 / 5} \\
& \times \frac{y^{1 / 5}}{\left(1+\operatorname{Pr}^{-1 / 2}\right)^{3 / 5}\left[\sin \left(2 \pi f_{n} \tau\right)\right]^{1 / 5} \mathrm{Ra}^{1 / 5}},
\end{aligned}
$$

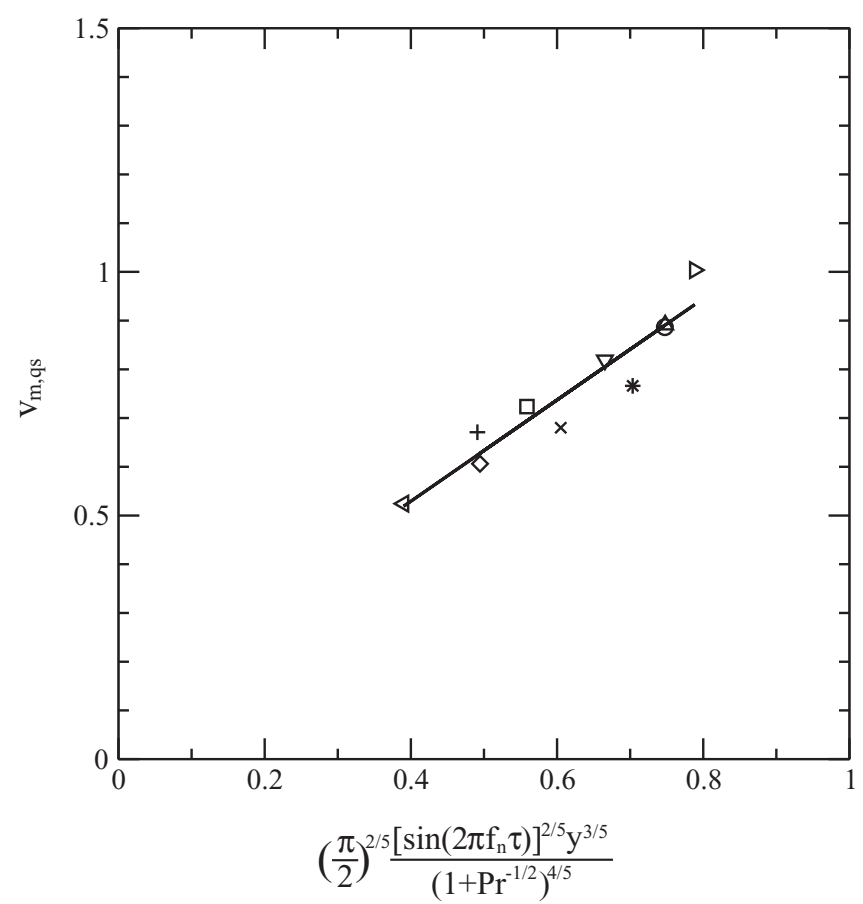

FIG. 12. Numerically obtained $v_{m, \mathrm{qs}}$ plotted against $(\pi / 2)^{2 / 5}$ $\left[\sin \left(2 \pi f_{n} \tau\right)\right]^{2 / 5} y^{3 / 5} /\left(1+\operatorname{Pr}^{-1 / 2}\right)^{4 / 5}$ for the 10 DNS runs. The solid line represents the linear correlation obtained by a linear curve-fitting based on the scaled data presented. The legend and its notations are the same as those in Fig. 10. 
which was obtained by a linear curve fitting. The regression coefficient of this best-fit line is 0.9929 , also confirming that the scaling (53) is in good agreement with the DNS results.

The scaling for the dimensionless maximum vertical velocity within the boundary layer at the quasisteady stage, $v_{m, \mathrm{qs}}$, is (51), which shows that $v_{m, \mathrm{qs}} \sim$ $(\pi / 2)^{2 / 5}\left[\sin \left(2 \pi f_{n} \tau\right)\right]^{2 / 5} y^{3 / 5} /\left(1+\operatorname{Pr}^{-1 / 2}\right)^{4 / 5}$, indicating that $v_{m, \mathrm{qs}}$ depends on $\operatorname{Pr}, f_{n}$, and $y$, but not on Ra, similar to the scaling (45) for $v_{m, s}$, which is the dimensionless maximum vertical velocity within the boundary layer at the transition time scale. The numerical verification of this scaling is demonstrated in Fig. 12, where the numerically obtained $v_{m \text {,qs }}$ is plotted against $(\pi / 2)^{2 / 5}\left[\sin \left(2 \pi f_{n} \tau\right)\right]^{2 / 5} y^{3 / 5} /\left(1+\operatorname{Pr}^{-1 / 2}\right)^{4 / 5}$ for the same 10 cases as those for Fig. 10 and Fig. 11. It is seen that all data presented in Fig. 12 fall reasonably well onto a straight line represented by

$$
v_{m, \mathrm{qs}}=0.1136+1.0393\left(\frac{\pi}{2}\right)^{2 / 5} \frac{\left[\sin \left(2 \pi f_{n} \tau\right)\right]^{2 / 5} y^{3 / 5}}{\left(1+\operatorname{Pr}^{-1 / 2}\right)^{4 / 5}},
$$

with the regression coefficient of 0.9492. It is observed that the DNS results at higher $\operatorname{Pr}\left(\operatorname{Pr}=50\right.$ and 100) and $f_{n}=$ 0.025 and 0.01 have noticeable deviations from the correlation represented by (65), due to the same reason as discussed in the above section.

The scaling for the dimensionless plate temperature at the quasi-steady stage, $\theta_{w, \mathrm{qs}}$, is (52), which shows that $\theta_{w, \mathrm{qs}} \sim$ $(\pi / 2)^{4 / 5} y^{1 / 5}\left(1+\operatorname{Pr}^{-1 / 2}\right)^{2 / 5}\left[\sin \left(2 \pi f_{n} \tau\right)\right]^{4 / 5} / \mathrm{Ra}^{1 / 5}$, indicating that $\theta_{w, \mathrm{qs}}$ depends on $\operatorname{Pr}, f_{n}, y$, and $\mathrm{Ra}$. This scaling is

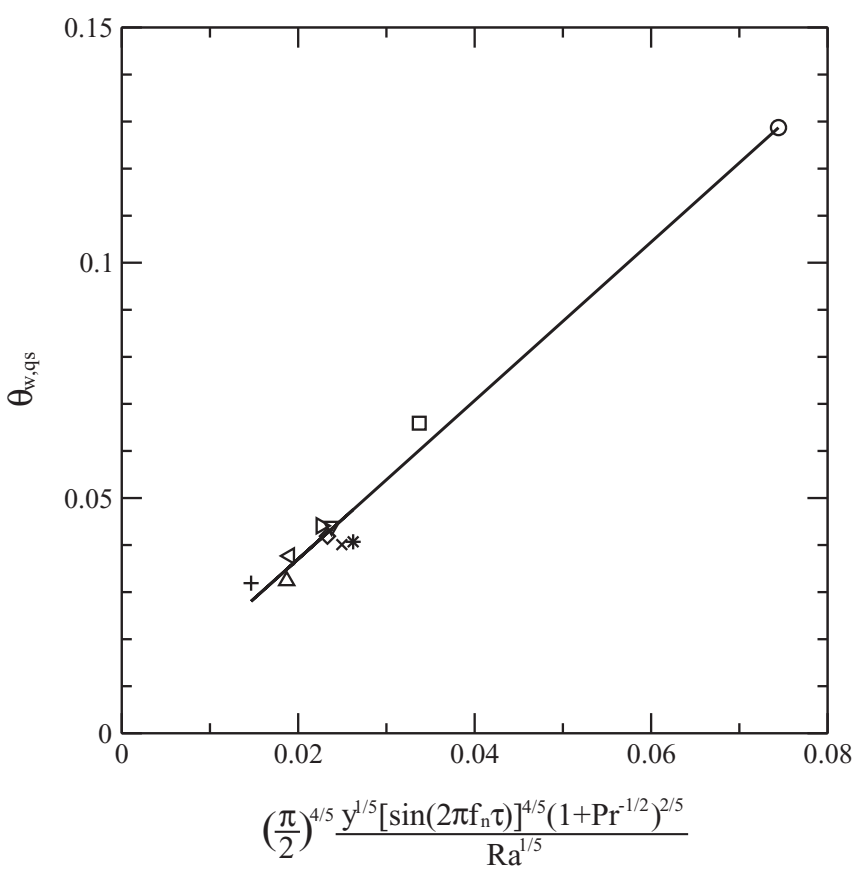

FIG. 13. Numerically obtained $\theta_{w, \text { qs }}$ plotted against $(\pi / 2)^{4 / 5}(1+$ $\left.\operatorname{Pr}^{-1 / 2}\right)^{2 / 5} y^{1 / 5}\left[\sin \left(2 \pi f_{n} \tau\right)\right]^{4 / 5} / \mathrm{Ra}^{1 / 5}$ for the 10 DNS runs. The solid line represents the linear correlation obtained by a linear curve-fitting based on the scaled data presented. The legend and its notations are the same as those in Fig. 10. also found to be verified by the numerical results, as shown in Fig. 13, where the numerically obtained $\theta_{w, \text { qs }}$ is plotted against $(\pi / 2)^{4 / 5} y^{1 / 5}\left(1+\operatorname{Pr}^{-1 / 2}\right)^{2 / 5}\left[\sin \left(2 \pi f_{n} \tau\right)\right]^{4 / 5} / \mathrm{Ra}^{1 / 5}$ for the same 10 cases as those for Figs. 10-12. It is seen that all data presented in Fig. 13 fall reasonably well onto a straight line represented by

$$
\begin{aligned}
\theta_{w, \mathrm{qs}}= & 0.0034+1.6842\left(\frac{\pi}{2}\right)^{4 / 5} \\
& \times \frac{y^{1 / 5}\left(1+\operatorname{Pr}^{-1 / 2}\right)^{2 / 5}[\sin (2 \pi f t)]^{4 / 5}}{\mathrm{Ra}^{1 / 5}},
\end{aligned}
$$

with the regression coefficient of 0.9906 . It is observed that the DNS results at $f_{n}=0.025$ and 0.01 have noticeable deviations from the correlation represented by (66).
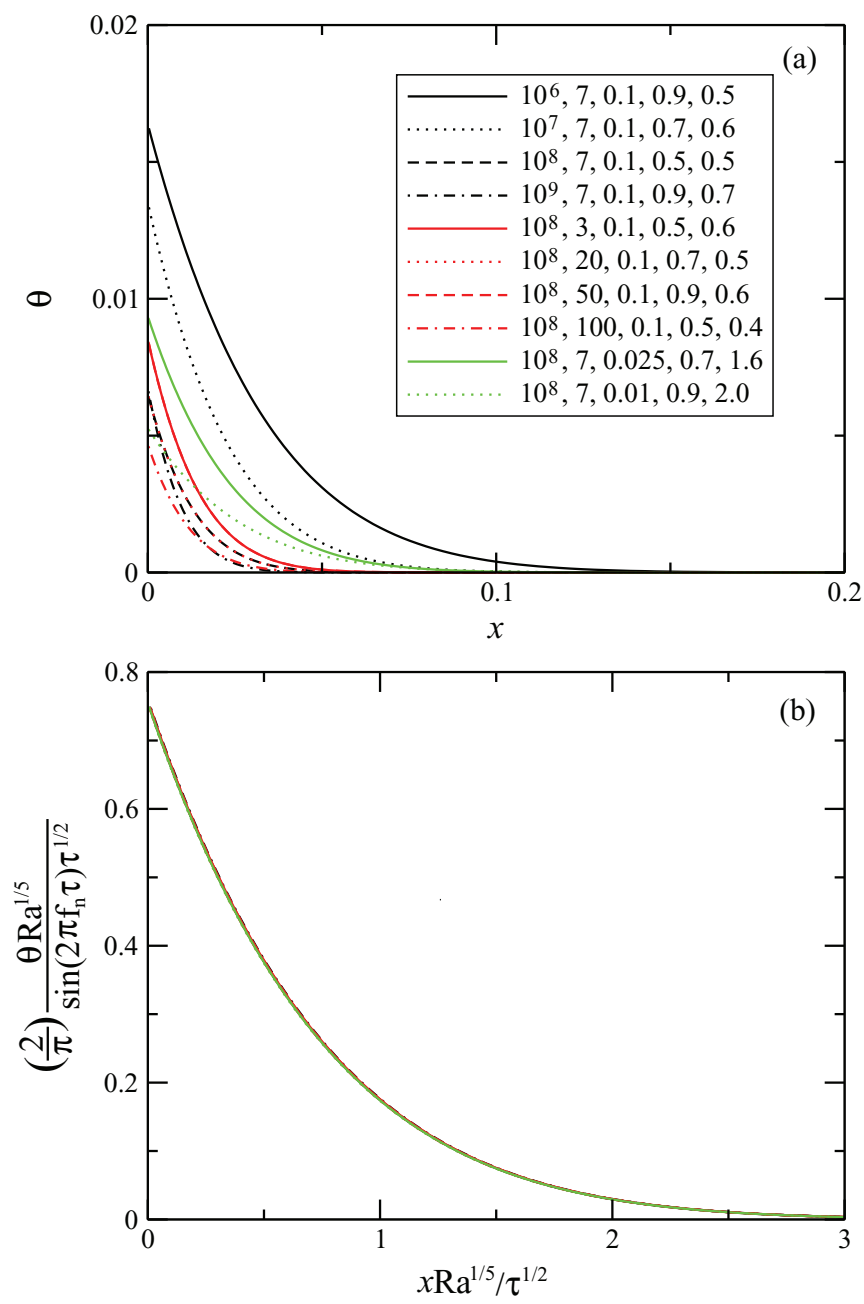

FIG. 14. (Color online) Numerically obtained horizontal temperature profiles at different heights for the 10 DNS runs at the start-up stage: (a) raw data and (b) scaled data, where $\theta$ is scaled by the scaling (39) and $x$ is scaled by the scaling (38), which are the scalings for $\theta_{w}$ and $\delta_{T}$ at the start-up stage, respectively. The notation in the legend in (a), e.g., $10^{8}, 7,0.1,0.5,0.5$ represents the DNS run of $\mathrm{Ra}=10^{8}$, $\operatorname{Pr}=7$, and $f_{n}=0.1$ at the height of $y=0.5$ and at the time instant $\tau=0.5$. 


\section{Scaled horizontal temperature and vertical velocity profiles}

The scalings (39) and (38) for $\theta_{w}$ and $\delta_{T}$ at the start-up stage can also be validated in terms of the scaled horizontal temperature profiles, as demonstrated in Fig. 14, where the raw data of the horizontal temperature profiles at different heights for the 10 DNS runs at the start-up stage, and their scaled forms, are presented. In Fig. 14(b), $\theta$ is scaled by the scaling (39) and $x$ is scaled by the scaling (38). From this figure, it is very evident that these two scalings collapse all scaled horizontal temperature profiles at the start-up stage onto the same curve.

Similar validations can also be made in terms of the scaled horizontal temperature profiles for the scalings for $\theta_{w, s}$ and $\delta_{T, s}$ at the transition time scale $\tau_{s}$. As shown in Fig. 15, where the raw data of the horizontal temperature profiles at different
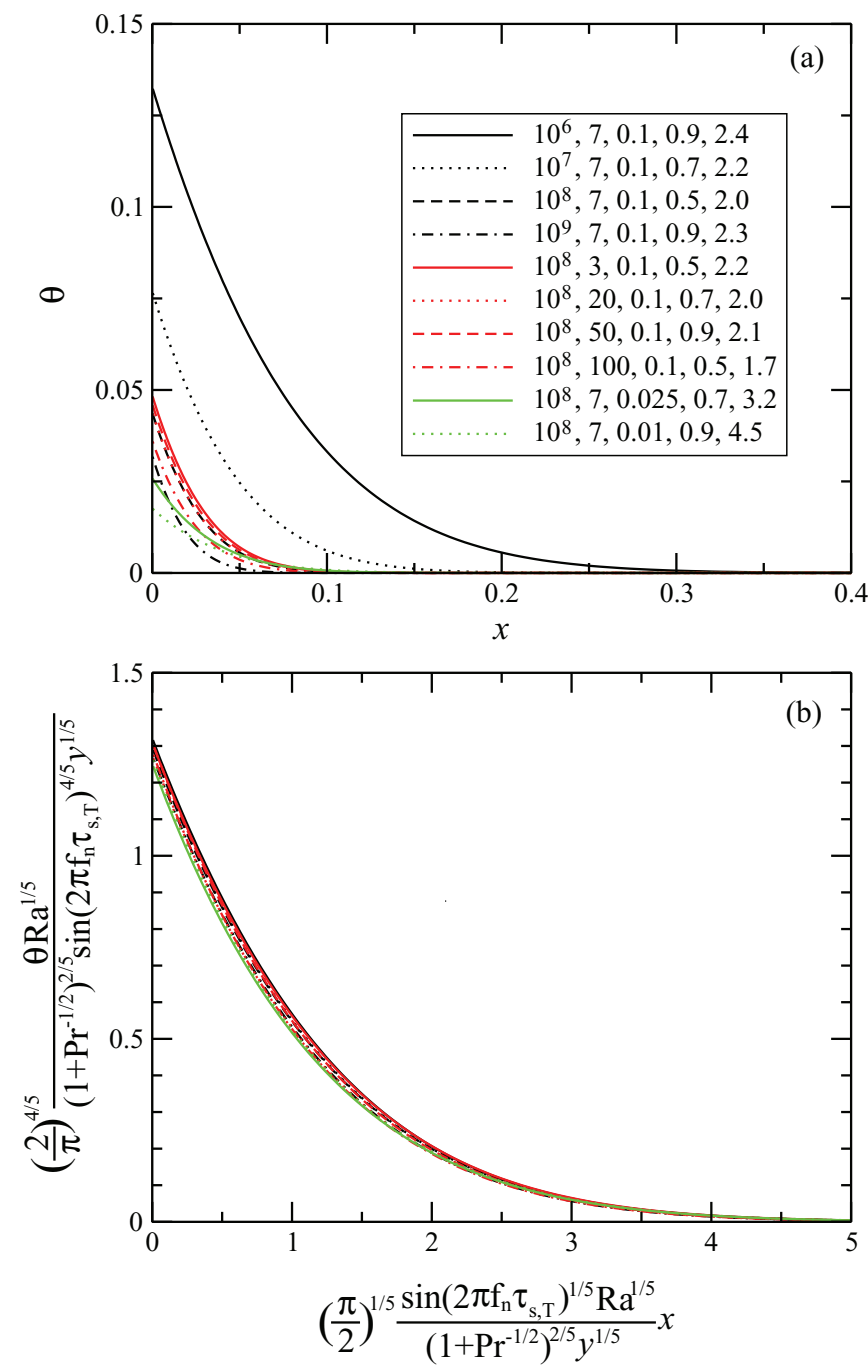

FIG. 15. (Color online) Numerically obtained horizontal temperature profiles at different heights for the $10 \mathrm{DNS}$ runs at the transition time scales: (a) raw data and (b) scaled data, where $\theta$ is scaled by the scaling (49) and $x$ is scaled by the scaling (46), which are the scalings for $\theta_{w}$ and $\delta_{T}$ at the transition time scales, respectively. The notation in the legend in (a), e.g., $10^{8}, 7,0.1,0.5,2.0$ represents the DNS run of $\operatorname{Ra}=10^{8}, \operatorname{Pr}=7$, and $f_{n}=0.1$ at the height of $y=0.5$ and at the time instant $\tau=2.0$.
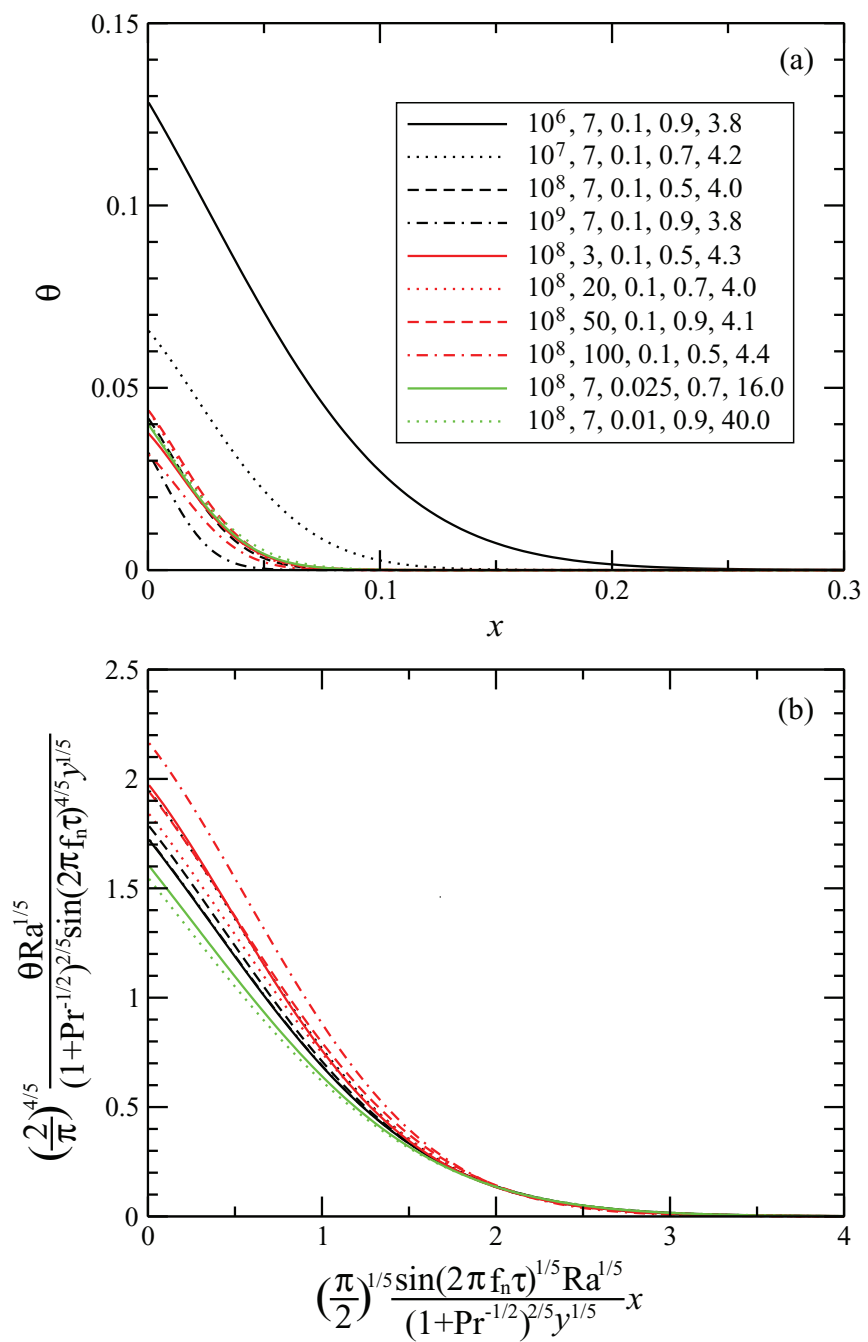

FIG. 16. (Color online) Numerically obtained horizontal temperature profiles at different heights for the 10 DNS runs at the quasi-steady stage: (a) raw data and (b) scaled data, where $\theta$ is scaled by the scaling (52) and $x$ is scaled by the scaling (50), which are the scalings for $\theta_{w}$ and $\delta_{T}$ at the quasi-steady stage, respectively. The notation in the legend in $(a)$, e.g., $10^{8}, 7,0.1,0.5,4.0$ represents the DNS run of $\operatorname{Ra}=10^{8}, \operatorname{Pr}=7$, and $f_{n}=0.1$ at the height of $y=0.5$ and at the time instant $\tau=4.0$.

heights for the 10 DNS runs at the transition time scale $\tau_{s}$ and their scaled forms are presented, the two scalings (49) and (46) bring all scaled horizontal temperature profiles almost into the same curve.

Figure 16 presents the raw data of the horizontal temperature profiles at different heights for the $10 \mathrm{DNS}$ runs at the quasi-steady stage and their scaled forms. In Fig. 16(b), the temperature $\theta$ and $x$ are scaled by $(\pi / 2)^{4 / 5} y^{1 / 5}\left(1+\operatorname{Pr}^{-1 / 2}\right)^{2 / 5}$ $\left[\sin \left(2 \pi f_{n} \tau\right)\right]^{4 / 5} / \mathrm{Ra}^{1 / 5}$ and $(2 / \pi)^{1 / 5}\left(1+\operatorname{Pr}^{-1 / 2}\right)^{2 / 5} y^{1 / 5} /$ $\mathrm{Ra}^{1 / 5} /\left[\sin \left(2 \pi f_{n} \tau\right)\right]^{1 / 5}$, respectively, the scalings for $\theta_{w, \mathrm{qs}}$ and $\delta_{T, \mathrm{qs}}$ at the quasi-steady stage, i.e., (52) and (50). It is apparent that the scaling (50) is in very good agreement with the numerical results as it brings all scaled temperature profiles together in the outer thermal boundary-layer region. Although the scaling (52) is found to be in a reasonable agreement with the numerical results, it does produce quite noticeable 

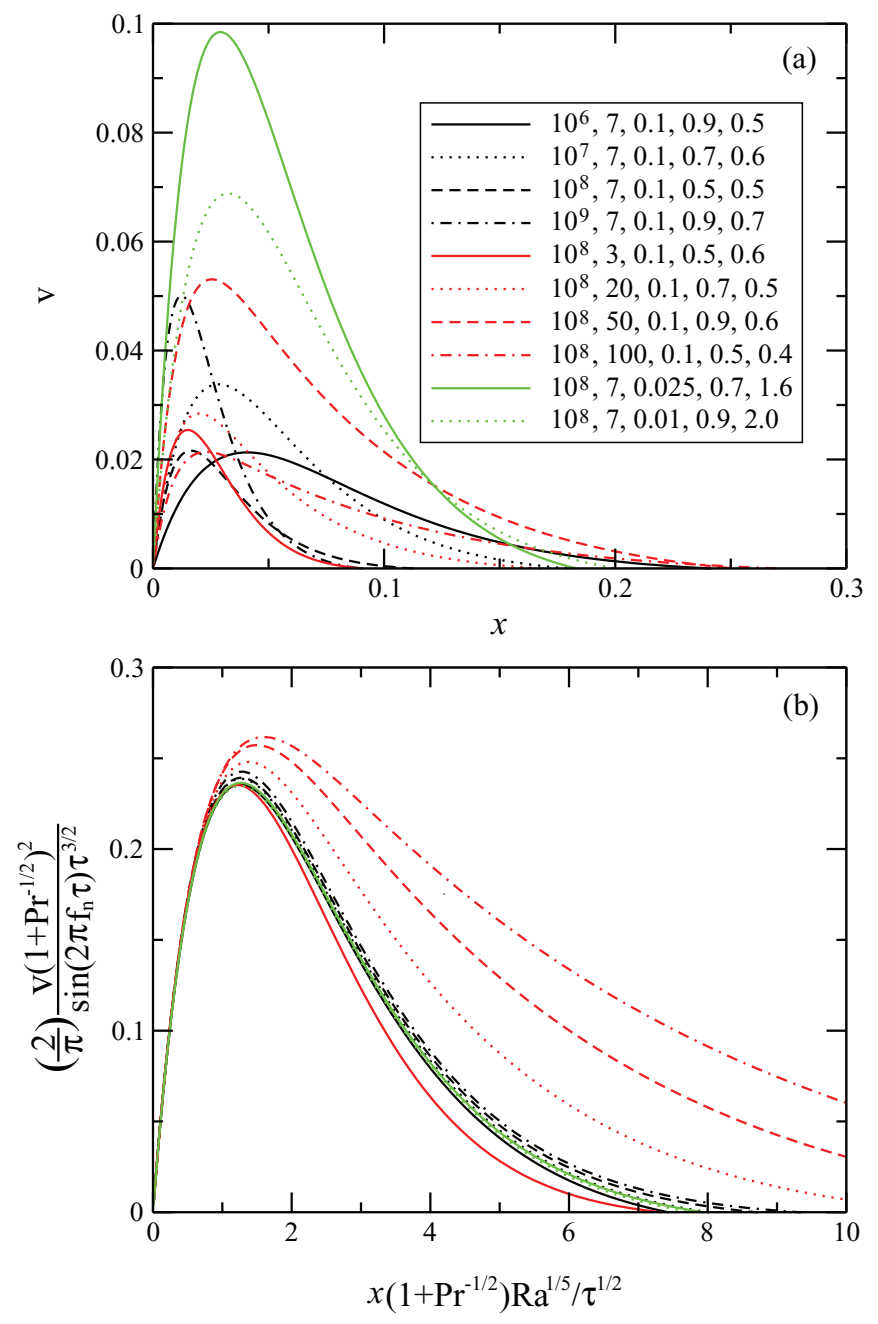

FIG. 17. (Color online) Numerically obtained horizontal profiles of vertical velocity at different heights for the 10 DNS runs at the start-up stage: (a) raw data and (b) scaled data, where $v$ is scaled by the scaling (41) and $x$ is scaled by the scaling (40), which are the scalings for $v_{m}$ and $\delta_{v i}$ at the start-up stage, respectively. The notation in the legend in (a), e.g., $10^{8}, 7,0.1,0.5,0.5$ represents the DNS run of $\mathrm{Ra}=10^{8}, \operatorname{Pr}=7$, and $f_{n}=0.1$ at the height of $y=0.5$ and at the time instant $\tau=0.5$.

deviations for higher- $\operatorname{Pr}$ cases $(\operatorname{Pr}=50$ and 100) and smaller $f_{n}$ cases $\left(f_{n}=0.025\right.$ and 0.01$)$, in the region close to the plate. The deviations for the higher-Pr cases are most likely due to the nonboundary nature of the flow, as discussed above.

Likewise, the scalings for $v_{m}$ and $\delta_{v i}$ at the start-up stage, at the transition time scale $\tau_{s}$, and at the quasi-steady stage, i.e., (41), (40), (45), (47), (51), and (53), can also be validated by the scaled horizontal profiles of vertical velocity within the inner viscous boundary layer (i.e., within region I), as shown in Fig. 17 for the start-up stage, in Fig. 18 for the transition time scale $\tau_{s}$, and in Fig. 19 for the quasi-steady stage. In Fig. 17, where the raw data of the numerically obtained horizontal profiles of vertical velocity at different heights for the 10 DNS runs at different time instants at the start-up stage and their scaled forms are presented, it is found that the scalings (41) and (40), for $v_{m}$ and $\delta_{v i}$ at the start-up stage, respectively,
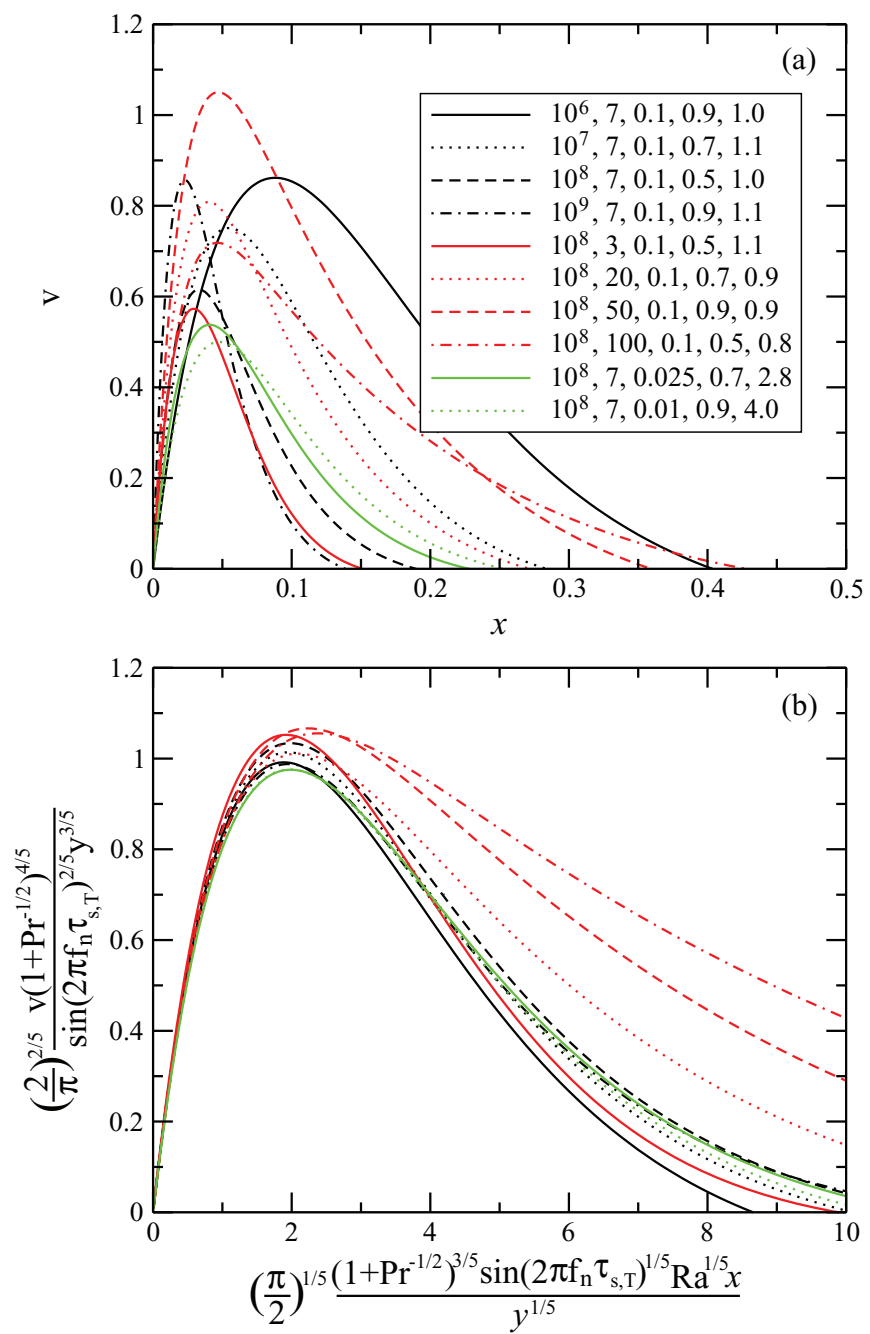

FIG. 18. (Color online) Numerically obtained horizontal profiles of vertical velocity at different heights for the 10 DNS runs at the transition time scales: $(a)$ raw data and $(b)$ scaled data, where $v$ is scaled by the scaling (45) and $x$ is scaled by the scaling (47), which are the scalings for $v_{m}$ and $\delta_{v i}$ at the transition time scales, respectively. The notation in the legend in $(a)$, e.g., $10^{8}, 7,0.1,0.5$, 1.0 represents the DNS run of $\mathrm{Ra}=10^{8}, \mathrm{Pr}=7$, and $f_{n}=0.1$ at the height of $y=0.5$ and at the time instant $\tau=1.0$.

collapse all scaled profiles onto the same curve within the inner viscous boundary layer (region I), which confirms these scalings. Similarly, in Fig. 18, where the raw data of the numerically obtained horizontal profiles of vertical velocity at different heights for the 10 DNS runs at their respective transition time scales $\tau_{s}$ and their scaled forms are presented, it is found that the scalings (45) and (47), for $v_{m}$ and $\delta_{v i}$, also collapse all scaled profiles onto the same curve within the inner viscous boundary layer (region I), which again confirms that these two scalings are correct. In Fig. 19, where the raw data of the numerically obtained horizontal profiles of vertical velocity at different heights for the 10 DNS runs at different time instants in the quasi-steady stage, and their scaled forms, are presented, it is found that the scalings (51) and (53), for $v_{m}$ and $\delta_{v i}$ at the quasi-steady stage, respectively, also collapse all 

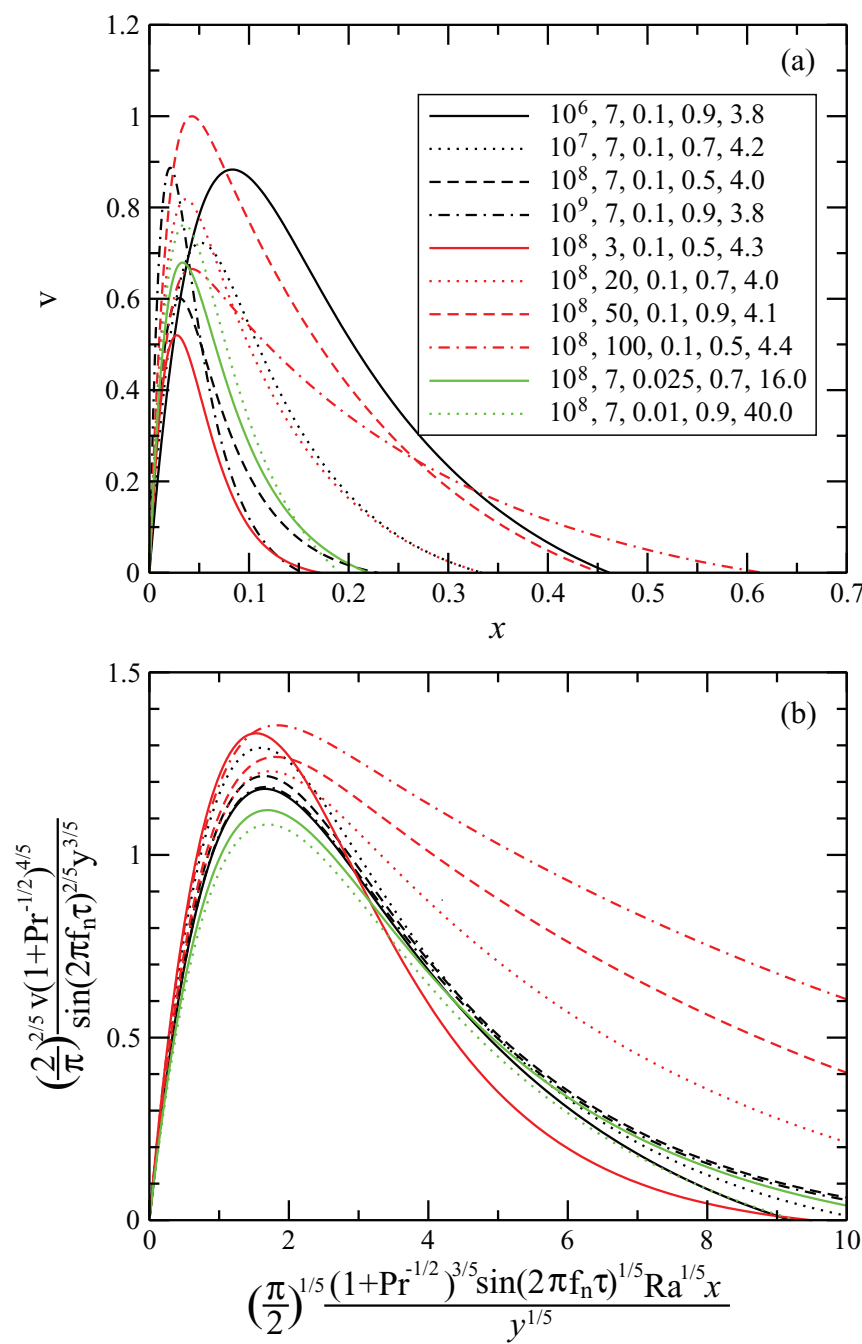

FIG. 19. (Color online) Numerically obtained horizontal profiles of vertical velocity at different heights for the 10 DNS runs at the quasi-steady stage: (a) raw data and (b) scaled data, where $v$ is scaled by the scaling (51) and $x$ is scaled by the scaling (53), which are the scalings for $v_{m}$ and $\delta_{v i}$ at the quasi-steady stage, respectively. The notation in the legend in (a), e.g., $10^{8}, 7,0.1,0.5,4.0$ represents the DNS run of $\mathrm{Ra}=10^{8}, \operatorname{Pr}=7$, and $f_{n}=0.1$ at the height of $y=0.5$ and at the time instant $\tau=4.0$.

scaled profiles onto the same curve within the inner viscous boundary layer (region I), which again confirms that these two scalings are the correct. All these three figures show that the scalings have quite noticeable deviations when Pr is higher than 7, due to the same limitations experienced by the current scaling analysis using the simple three-region structure as discussed above, and when $f_{n}$ is reduced, which again requires a further investigation into the possible additional dependence on $f_{n}$.

\section{CONCLUSIONS}

In this paper, it was found that the transient and quasi-steady flow behavior of the unsteady natural convection boundary layer of a homogeneous Newtonian fluid with $\operatorname{Pr}>1$ adjacent to a vertical plate evenly heated with a time-varying sinusoidal heat flux is controlled by the Rayleigh number $\mathrm{Ra}$, the Prandtl number Pr, and the dimensionless natural frequency $f_{n}$ of the sinusoidal heat flux and is well represented by parameters such as the thermal boundary-layer thickness, the plate temperature, the viscous boundary-layer thickness, and the maximum vertical velocity within the boundary layer. Scalings were developed using a simple three-region structure proposed by Refs. [41,42] for the flow and were compared with a series of direct numerical simulation, which clearly shows that these scalings in general predict the flow behavior very well, no matter at the start-up stage, the transition time scale which represents the end of the start-up stage and the beginning of the transitional stage of the boundary-layer development, or the quasi-steady stage. However, noticeable deviations were observed for high-Pr fluids which indicates that a further, although weak, Pr dependence is present and is speculated to be caused by the non-boundary-layer nature of the velocity field for high-Pr fluids. Some scalings at the transition time scale and at the quasi-steady stage also show additional $f_{n}$ dependence for small $f_{n}$. Better and more accurate scaling approximations should be investigated to predict such a further Pr dependence in high-Pr fluids and any possible further $f_{n}$ dependence for the flow; however, this is beyond the scope of the current study.

\section{ACKNOWLEDGMENTS}

Support from the National Natural Science Foundation of China (Grant No. 11072211), the Natural Science Foundation of Yunnan Province (2011FA017), the Program for Changjiang Scholars and Innovative Research Team in University of Ministry of Education of China, and the Australian Research Council is gratefully acknowledged.
[1] J. C. Patterson and J. Imberger, J. Fluid Mech. 100, 65 (1980).

[2] W. Lin and S. W. Armfield, Phys. Rev. E 86, 066312 (2012).

[3] J. A. Duffie and W. A. Beckman, Solar Engineering of Thermal Processes, 3rd ed. (Wiley, New York, 2006).

[4] R. B. Yedder and E. Bilgen, Int. J. Heat Mass Transf. 34, 1237 (1991).

[5] S. A. M. Burek and A. Habeb, Energ. Buildings 39, 128 (2007).

[6] A. Y. K. Tan and N. H. Wong, Energ. Buildings 53, 19 (2012).
[7] O. Saadatian, K. Sopian, C. H. Lim, N. Asim, and M. Y. Sulaiman, Renew. Sust. Energ. Rev. 16, 6340 (2012).

[8] C. Li and R. Z. Wang, Renew. Sust. Energ. Rev. 16, 6191 (2012).

[9] P. Cheng, Lett. Heat Mass Transf. 4, 119 (1977).

[10] V. P. Carey and J. C. Mollendorf, Int. J. Heat Mass Transf. 21, 481 (1978).

[11] E. M. Sparrow and J. L. Gregg, Trans. Am. Soc. Mech. Engrs. 78, 435 (1956)

[12] A. Bejan, Int. J. Heat Mass Transf. 26, 1339 (1983). 
[13] F. H. Bark, F. Alavyoon, and A. A. Dahlkild, J. Fluid Mech. 235, 665 (1992).

[14] F. Alavyoon, Int. J. Heat Mass Transf. 36, 2479 (1993).

[15] H. E. Imadojemu and R. R. Johnson, Exp. Therm. Fluid Sci. 9, 13 (1994).

[16] L. G. Sundström and M. Vynnycky, J. Fluid Mech. 390, 45 (1999).

[17] O. Aydin and L. Guessous, Int. J. Heat Mass Transf. 44, 4605 (2001).

[18] S. W. Armfield, J. C. Patterson, and W. Lin, Int. J. Heat Mass Transf. 50, 1592 (2007).

[19] W. Lin, S. W. Armfield, and J. C. Patterson, Int. J. Heat Mass Transf. 51, 327 (2008).

[20] T. P. Bednarz, C. Lei, and J. C. Patterson, Int. J. Heat Mass Transf. 52, 56 (2009).

[21] W. A. Khan and A. Aziz, Int. J. Thermal Sci. 50, 1207 (2011).

[22] M. Capobianchi and A. Aziz, Int. J. Therm. Sci. 54, 82 (2012).

[23] S. C. Saha, R. J. Brown, and Y. T. Gu, Int. J. Heat Mass Transf. 55, 2394 (2012).

[24] B. Buonomo and O. Manca, Int. J. Therm. Sci. 56, 35 (2012).

[25] J. H. Merkin, European J. Mech. B/Fluids 36, 75 (2012).

[26] P. Dutta and K. N. Seetharamu, Wärme- und Stoffübertragung 28, 27 (1993).

[27] H. Kou and D. Huang, Int. Comm. Heat Mass Transf. 23, 1031 (1996).

[28] S. D. Wright, D. B. Ingham, and I. Pop, Transp. Porous Media 22, 181 (1996).

[29] P. Ganesan and H. P. Rani, Heat Mass Transf. 35, 259 (1999).
[30] B. Tashtoush and E. Abu-Irshaid, Acta Mech. 152, 1 (2001).

[31] Shalini and B. V. R. Kumar, Comm. Nonlin. Sci. Numer. Simul. 12, 1454 (2007).

[32] R. Tsai and J. S. Huang, Comput. Mater. Sci. 47, 23 (2009).

[33] N. Mustafa, S. Asghar, and M. A. Hossain, Int. J. Heat Mass Transf. 53, 5856 (2010).

[34] G. Palani and K. Y. Kim, Appl. Math. Mech. 33, 605 (2012).

[35] J. Fohr and H. B. Moussa, Int. J. Heat Mass Transf. 37, 1699 (1994).

[36] Q. W. Wang, J. Yang, M. Zeng, and G. Wang, Int. J. Heat Fluid Flow 31, 70 (2010).

[37] G. Wang, M. Zeng, Y. C. Ren, H. Ozoe, and Q. W. Wang, Exp. Therm. Fluid Sci. 35, 105 (2011).

[38] S. W. Armfield and J. C. Patterson, J. Fluid Mech. 239, 195 (1992).

[39] W. Schopf and J. C. Patterson, Int. J. Heat Mass Transf. 39, 3497 (1996).

[40] W. Lin, S. W. Armfield, and J. C. Patterson, J. Fluid Mech. 574, 85 (2007).

[41] W. Lin, S. W. Armfield, J. C. Patterson, and C. Lei, Phys. Rev. E 79, 066313 (2009).

[42] J. C. Patterson, C. Lei, S. W. Armfield, and W. Lin, Int. J. Therm. Sci. 48, 1843 (2009).

[43] W. Lin and S. W. Armfield, Int. J. Heat Mass Transf. 42, 4117 (1999).

[44] W. Lin and S. W. Armfield, Int. J. Heat Fluid Flow 22, 72 (2001).

[45] W. Lin and S. W. Armfield, Phys. Rev. E 72, 016306 (2005).

[46] W. Lin and S. W. Armfield, Phys. Rev. E 72, 066309 (2005). 